\title{
A Cognition-Related Neural Oscillation Pattern, Generated in the Prelimbic Cortex, Can Control Operant Learning in Rats
}

\author{
(i)Samuel Hernández-González, ${ }^{1}$ Celia Andreu-Sánchez, ${ }^{2}$ Miguel Ángel Martín-Pascual, ${ }^{2}{ }^{\circledR}$ Agnès Gruart, ${ }^{1}$ \\ and ${ }^{\circ} J o s e ́$ María Delgado-García ${ }^{1}$ \\ ${ }^{1}$ Division of Neurosciences, Pablo de Olavide University, 41013 Seville, Spain, and ${ }^{2}$ Audiovisual Communication and Advertising Department, Universitat \\ Autònoma de Barcelona, 08193 Bellaterra, Spain
}

\begin{abstract}
The prelimbic (PrL) cortex constitutes one of the highest levels of cortical hierarchy dedicated to the execution of adaptive behaviors. We have identified a specific local field potential (LFP) pattern generated in the PrL cortex and associated with cognition-related behaviors. We used this pattern to trigger the activation of a visual display on a touch screen as part of an operant conditioning task. Rats learned to increase the presentation rate of the selected $\theta$ to $\beta-\gamma(\theta / \beta-\gamma)$ transition pattern across training sessions. The selected LFP pattern appeared to coincide with a significant decrease in the firing of PrL pyramidal neurons and did not seem to propagate to other cortical or subcortical areas. An indication of the PrL cortex's cognitive nature is that the experimental disruption of this $\theta / \beta-\gamma$ transition pattern prevented the proper performance of the acquired task without affecting the generation of other motor responses. The use of this LFP pattern to trigger an operant task evoked only minor changes in its electrophysiological properties. Thus, the PrL cortex has the capability of generating an oscillatory pattern for dealing with environmental constraints. In addition, the selected $\theta / \beta$ - $\gamma$ transition pattern could be a useful tool to activate the presentation of external cues or to modify the current circumstances.
\end{abstract}

Key words: behaving rats; brain-machine interaction; local field potentials; neural oscillations; operant conditioning; prelimbic cortex

Significance Statement

Brain-machine interfaces represent a solution for physically impaired people to communicate with external devices. We have identified a specific local field potential pattern generated in the prelimbic cortex and associated with goal-directed behaviors. We used the pattern to trigger the activation of a visual display on a touch screen as part of an operant conditioning task. Rats learned to increase the presentation rate of the selected field potential pattern across training. The selected pattern was not modified when used to activate the touch screen. Electrical stimulation of the recording site prevented the proper performance of the task. Our findings show that the prelimbic cortex can generate oscillatory patterns that rats can use to control their environment for achieving specific goals.

\section{Introduction}

The use of the neural cortical activity for operant conditioning tasks goes back to the 1970s, with the innovative approaches of Fetz and colleagues (Fetz, 1969; Fetz and Finocchio, 1971, 1972).

\footnotetext{
Received Nov. 28, 2016; revised March 25, 2017; accepted April 2, 2017

Author contributions: A.G. and J.M.D.-G. designed research; S.H.-G. performed research; C.A.-S. and M.Á.M.-P. contributed unpublished reagents/analytic tools; S.H.-G., C.A.-S., M.A.M.-P., and A.G. analyzed data; S.H.-G., A.G., and J.M.D.-G. wrote the paper.

This work was supported by grants from the Spanish Minesterio de Economia, Industría y Competitividad (MINECO; BFU2014-56692-R), the Junta de Andalucía (BI0122, CVI 2487, and P07-CVI-02686), and the Spanish Tatiana Pérez de Guzmán el Bueno Foundation to A.G. and J.M.D.-G.S.H.-G. was supported by a fellowship from the Spanish MINECO (BES-2012-052069). We thank J.A. Santos-Naharro for his help in instrumental design and analytical procedures and R. Churchill for proofreading.

The authors declare no competing financial interests.

Correspondence should be addressed to Professor José M. Delgado-García, División de Neurociencias, Universidad Pablo de Olavide, 41013-Seville, Spain. E-mail: jmdelgar@upo.es.

DOI:10.1523/JNEUROSCI.3651-16.2017

Copyright $\odot 2017$ the authors $\quad 0270-6474 / 17 / 375923-13 \$ 15.00 / 0$
}

Fetz's group used awake monkeys to demonstrate the conditioning of single neurons by reinforcing a high rate of neuronal activity with the delivery of a reward. Latterly, it was also shown that the information contained in local field potentials (LFPs) possesses features suited (Pesaran et al., 2002; Mehring et al., 2003) for use as brain-machine interfaces (BMIs; Flint et al., 2013; So et al., 2014). LFPs are more stable than unitary recordings and present higher spatial resolution and signal amplitudes than electroencephalographic recordings (Freeman et al., 2003; Shain et al., 2003).

Many studies have used the neural activity recorded in the M1 and/or the posterior parietal cortices for different experimental designs, including the activation of external devices (Lebedev and Nicolelis, 2006; Bouton et al., 2016). But, in the past few years, the appearance of noninvasive techniques has allowed the use of deep brain regions, such as the prefrontal cortex, for BMIs controlled by cognitive activities (Schudlo et al., 2013; Hong et al., 2015). BMIs have been largely explored from the point of view of inter- 
A

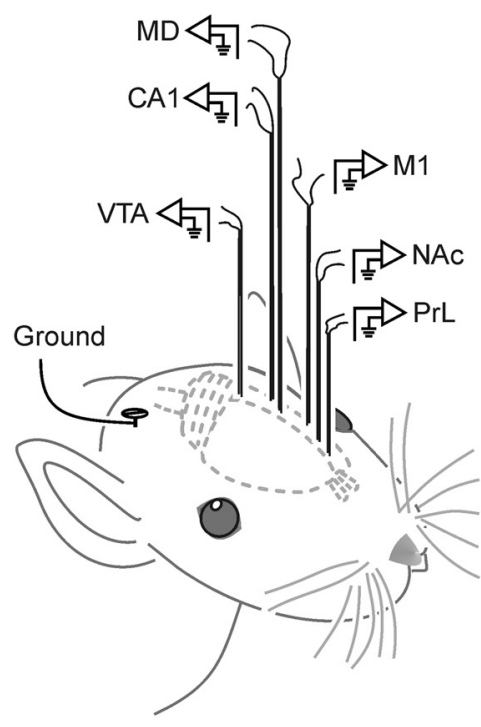

B

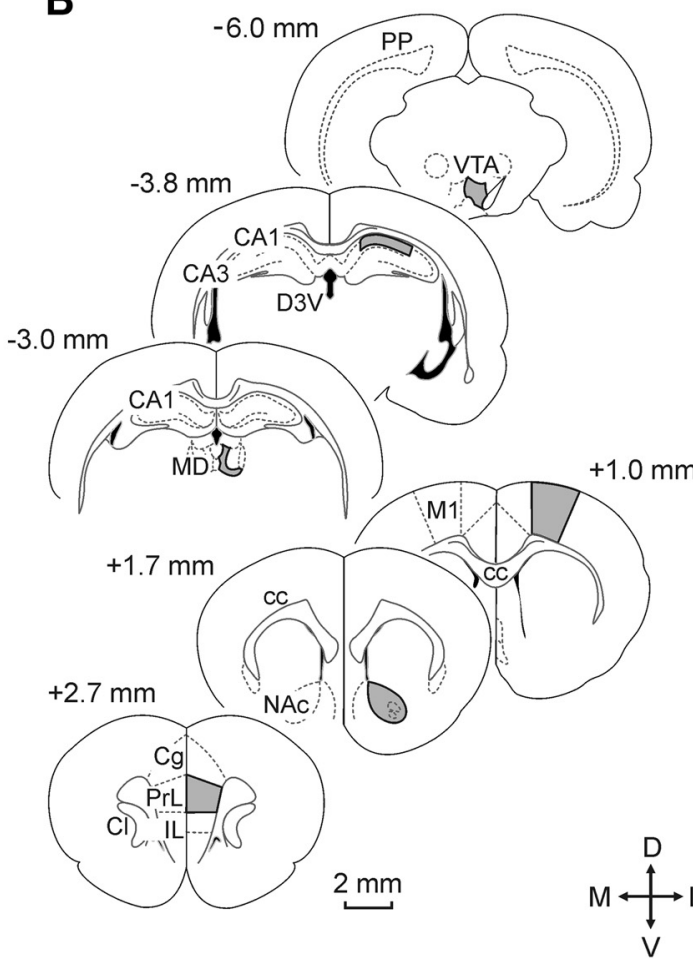

C

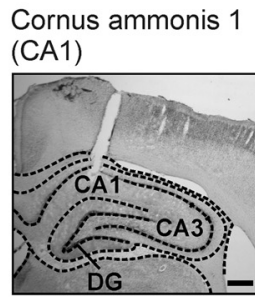

Mediodorsal thalamus (MD)

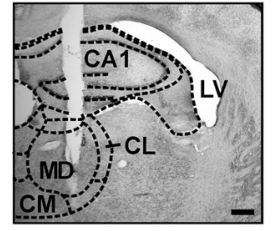

Prelimbic cortex (PrL)

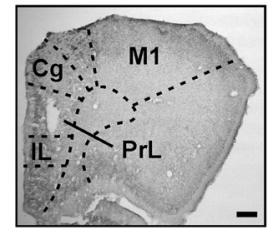

Ventral tegmental area (VTA)

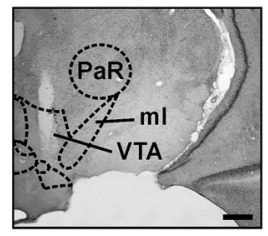

Primary motor cortex (M1)

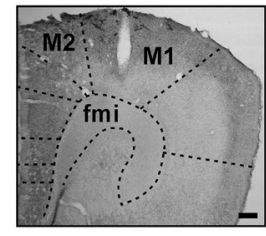

Nucleus accumbens (NAc)

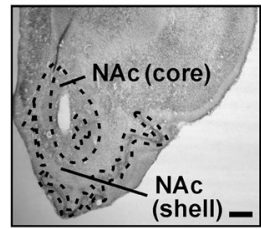

Figure 1. Experimental design for brain stimulation and LFP recordings. $\boldsymbol{A}$, Schematic representation of the location of stimulating and recording electrodes. $\boldsymbol{B}$, Diagrams of brain sagittal sections illustrating in gray the implanted cortical and subcortical areas. Coordinates with respect to bregma are indicated. $C$, Representative photomicrographs illustrating the location of implanted electrodes. Cg, Cingulate cortex; IL, infralimbic cortex; CC, corpus callosum; Cl, claustrum; CL, centrolateral thalamic nucleus; CM, central medial thalamic nucleus; D3V, dorsal third ventricle; DG, dentate gyrus; fmi, forceps minor of the corpus callosum; LV, lateral ventricle; ml, medial lemniscus; PaR, pararubral nucleus; PP, perforant pathway; D, dorsal; L, lateral; M, medial; V, ventral.

face design, including algorithms, motor imagery, and adapted electronic devices, but rarely from an electrophysiological approach, specifically a learning process where two events-a specific cortical activity pattern and a subsequent response generated in an external device-are progressively associated. In this regard, recent papers have been focused on the study of neural mechanisms underlying motor and cognitive processes using these sorts of interfaces (Koralek et al., 2012; Rouse et al., 2013; Wander et al., 2013). These types of study could help identify some of the physiological processes related to the actual motor and cognitive learning tasks (O'Doherty et al., 2011; Schafer and Moore, 2011; Hiremath et al., 2015).

Although they present many experimental (extended laboratory use, adequate brain size, easy acquisition of operant condition tasks) and ethical advantages, rats have not often been used in studies related to BMIs. In our approach, we have used a well defined LFP [a $\theta$ to $\beta-\gamma(\theta / \beta-\gamma)$ ] transition pattern recorded at the rostral prelimbic (PrL) cortex of rats to trigger the presentation of a visual display on an iPad tablet. The rat was rewarded with a pellet of food when touching the iPad screen. This operant conditioning design has the advantage of using a LFP pattern generated in a brain area not directly related to motor control commands (Khanna and Carmena, 2015), but to volitional and valuation processes (Kable and Glimcher, 2009; Graybeal et al., 2011; Fuster, 2015). We also studied the putative changes in the profiles of the LFPs used to activate the touch screen as well as the firing activities of putative PrL pyramidal cells to determine their contribution to the generation of the selected LFP pattern. In addition, we checked the cognitive nature of the selected LFP pattern by determining the animal's behavior associated with it and the behavioral effects of PrL cortex stimulation during the pattern's spontaneous appearances. In this regard, it has been recently described that the electrical stimulation of the PrL cortex recording sites prevent the proper performance of acquired operant conditioning tasks (Jurado-Parras et al., 2012). In summary, present results indicate that PrL cortex circuits seem to evoke specific oscillatory patterns probably related to attentional and cognitive processes that can be safely used to trigger external cues and/or to deal with specific environmental constrains.

\section{Materials and Methods}

Experimental animals. Experiments were performed on male Lister hooded rats (5-6 months old) provided by an authorized supplier (Charles River Laboratories). Upon arrival at the Pablo de Olavide Animal House (Seville, Spain), animals were housed in individual cages until the end of the experiment. Rats were kept on a $12 \mathrm{~h}$ light/dark cycle with constant ambient temperature $\left(21.5 \pm 1{ }^{\circ} \mathrm{C}\right)$ and humidity $(55 \pm 8 \%)$, with food and water available ad libitum. All experiments were performed in accordance with the guidelines of the European Union Council (2010/276:33-79/EU) and Spanish regulations (BOE 34:11370-421, 2013) for the use of laboratory animals in chronic studies, and approved by the local Ethics Committee of the Pablo de Olavide University.

Surgery. Animals were anesthetized with 1-2.5\% isoflurane delivered by a rat anesthesia mask (David Kopf Instruments). The anesthetic gas was supplied from a calibrated Fluotec 5 (Fluotec-Ohmeda) vaporizer, at a flow rate of $1-3 \mathrm{l} / \mathrm{min}$ oxygen (AstraZeneca). For LFP recordings (Fig. $1 B, C$ ), and following the Paxinos and Watson atlas (1998; RRID: SCR_006369), animals $(n=10)$ were chronically implanted with recording electrodes aimed at the PrL cortex ( $2.7 \mathrm{~mm}$ anterior to bregma, 0.8 $\mathrm{mm}$ lateral to bregma, and $3.6 \mathrm{~mm}$ below the brain surface), the nucleus accumbens ( $1.7 \mathrm{~mm}$ anterior to bregma, $1.4 \mathrm{~mm}$ lateral to bregma, and $7.6 \mathrm{~mm}$ below the brain surface), the primary motor cortex $(1.0 \mathrm{~mm}$ anterior to bregma, $2.3 \mathrm{~mm}$ lateral to bregma, and $2.0 \mathrm{~mm}$ below the brain surface), the mediodorsal thalamic nucleus ( $3.0 \mathrm{~mm}$ posterior to 
bregma, $0.5 \mathrm{~mm}$ lateral to bregma, and $5.3 \mathrm{~mm}$ below the brain surface), the hippocampal CA1 area (3.8 $\mathrm{mm}$ posterior to bregma, $2.0 \mathrm{~mm}$ lateral to bregma, and $2.5 \mathrm{~mm}$ below the brain surface), and the ventral tegmental area $(6.0 \mathrm{~mm}$ posterior to bregma, $0.5 \mathrm{~mm}$ lateral to bregma, and 7.8 $\mathrm{mm}$ below the brain surface). These electrodes were made from $50 \mu \mathrm{m}$, Teflon-coated, tungsten wire (Advent Research). Each electrode consisted of three individual tungsten wires with their tips spaced $300 \mu \mathrm{m}$ apart along the extent of the electrode. With the aim of recording the unitary activity present in the PrL cortex during the appearance of the selected $\theta / \beta-\gamma$ transition pattern, five additional animals were implanted in the PrL cortex with the same recording electrode but with the addition of a home-made multielectrode. The multielectrode was composed of six $25 \mu \mathrm{m}$, Teflon-coated, tungsten wires (Advent Research).

To determine whether vibrissal movements were related to LFPs recorded at the PrL cortex, a pair of recording electrodes was implanted in the center of the contralateral whisker pad in the above-mentioned animals. Vibrissal electrodes were made from $50 \mu \mathrm{m}$, Teflon-coated, annealed stainless steel wire (A-M Systems). A bare silver wire was affixed to the bone as ground. All the implanted wires were soldered to three sixpin sockets (RS Amidata) and fixed to the skull with dental cement (Fig. 1; Gruart et al., 2006).

Since the presence of recording wires could represent a significant impairment for the proper acquisition of the pattern-triggered tasks, an additional group $(n=5)$ of rats were chronically implanted with five 50 $\mu \mathrm{m}$ tungsten recording electrodes aimed at the PrL cortex. These electrodes, plus a ground wire, were connected to a Bakelite integrated circuit (Cibertec) equipped with an eight-pin Omnetics socket (RS Amidata).

Recording and stimulating procedures. LFP and electromyographic recordings were performed using Grass P511 differential amplifiers with a bandwidth of $0.1-10 \mathrm{kHz}$ (Grass-Telefactor). Wireless recordings were performed with the help of a five-channel TBSI head-stage system (Harvard Bioscience). Unitary recordings were performed with high-impedance probes $\left(2 \times 10^{12} \Omega, 10 \mathrm{pF}\right)$ and filtered between 450 and $4500 \mathrm{~Hz}$, with the help of a finite impulse response filter.

For the electrical stimulation of the PrL cortex region during operant conditioning, we used a $200 \mathrm{~Hz}$ train of pulses (50 $\mu$ s negative-positive) lasting $200 \mathrm{~ms}$. The train was triggered, with a delay of $<0.5 \mathrm{~ms}$, by a touch of a square virtual button on the iPad screen. To avoid excessive stimulations of the experimental animal, subsequent iPad touches within a $6 \mathrm{~s}$ window did not trigger any stimulus. Stimulus intensities ranged from 0.5 to $1 \mathrm{~mA}$. For each animal, we selected the minimum intensity evoking a short disruption in the expected behavioral sequence (touch the visual display, go to the feeder, eat the pellet; Jurado-Parras et al., 2012).

Instrumental conditioning. In a preliminary series of experiments, a group of rats $(n=5)$ were trained for operant conditioning in a basic Skinner box module measuring $29.2 \times 24.1 \times 21 \mathrm{~cm}$ (MED Associates). The operant chamber was housed within a sound-attenuating chamber $(90 \times 55 \times 60 \mathrm{~cm})$, which was constantly illuminated $(19 \mathrm{~W}$ lamp $)$ and exposed to a $45 \mathrm{~dB}$ of white noise (Cibertec). The Skinner box was equipped with a food dispenser from which pellets (Noyes formula P; 45 $\mathrm{mg}$; Sandown Scientific) could be delivered by pressing a lever. Before training, rats were handled daily for $>7 \mathrm{~d}$ and food-deprived to $80-85 \%$ of their ad libitum feeding weight. Once the desired weight was reached, animals were placed in the Skinner box for $20 \mathrm{~min}$ and allowed to press the lever to receive pellets from the food tray using a fixed-ratio (FR 1:1) schedule, until reaching criterion. Criterion was to press the lever $>80$ times/session for 2 consecutive days (Fig. 2; Hasan et al., 2013). Another group of rats $(n=5)$ were trained in a modified Skinner box module of similar dimensions (MED Associates) equipped with an iPad tablet computer and the same food dispenser from which pellets could be delivered by touching a square $(200 \times 200$ pixels; i.e., $\approx 3.4 \times 3.4 \mathrm{~cm})$ virtual button displayed on the screen. In this case, rats were pretreated and trained as indicated above. Collected results of this comparative study are illustrated in Figure $2 B, C$.

Conditioning programs, LFP pattern detections, lever presses, screen touches, and delivered reinforcements were monitored and recorded with a computer, using a MED-PC program (MED Associates; RRID: SCR_012156). All operant sessions were recorded with a video capture system (Sony HDR-SR12E) synchronized to LFP recordings.
Activation of the touch screen by LFPs recorded in the PrL cortex. In the next experiments, rats implanted with recording electrodes were trained to activate the visual display on the touch screen by LFP patterns recorded from the PrL cortex. For this, a capacitive multitouch-screen device (iPad 2, Apple) with a 9.7 inch LED-back-lit glossy widescreen display with in-plane switching technology (Leising et al., 2013) was used. It had $1024 \times 768$-pixel resolution at 132 pixels per inch.

First, rats were trained to touch the square virtual button in the modified Skinner box until reaching the above-specified criterion. Then, they had to learn to trigger the appearance of the square button on the touch screen by the generation of a $\theta / \beta-\gamma$ transition pattern. This LFP pattern was identified in previous recordings of PrL cortex activities as being related to the presentation of behaviors, such as remaining still (motionless) in the cage, observing the feeder, or rearing (Figs. 3, 4). This specific LFP pattern was characterized by a significant decrease in power in the $\theta$ band (Fig. $3 A, B$ ) accompanied by smaller changes in the power of both $\beta$ and $\gamma$ bands (Fig. $3 E$ ). The detection of this $\theta / \beta-\gamma$ transition pattern was based on the instantaneous calculation of the power in a wide band of frequencies between 3 and $150 \mathrm{~Hz}$ that included the three mentioned oscillation bands $(\theta, \beta$, and $\gamma)$. This spectral analysis was performed with the help of a home-made program implemented with Spike2 software (CED 1401 Plus, Cambridge Electronics Design, RRID:SCR_000903). Power was calculated using the fast Fourier transform with a raised cosine window. In addition to the above, a spectral power threshold was calculated from LFPs recorded during preliminary baseline and training sessions and maintained during the whole experiment. According to the intrinsic characteristics of the LFP recordings from each experimental rat, the criterion values assigned to the threshold ranged from 0.00125 to $0.00215 \mathrm{~V}^{2}$. Thresholds were calculated as the average of the minimum values of power between 3 and $150 \mathrm{~Hz}$ plus $3 \times \mathrm{SD}$. The detections of the selected $\theta / \beta-\gamma$ transition pattern generated a new event channel in Spike2, which was translated into a command system to display the visual stimulus on the touch screen (Fig. 5A).

For the command system, we developed an ad hoc software (RatButton 2.1.3. for iOS 8.4) for stimulus presentation on the touch screen triggered by the selected $\theta / \beta-\gamma$ transition pattern. The RatButton system was activated when power for the three bands fell below the established power threshold. The same software also activated the feeder to supply the reward and quantify the number and time of correct screen touches. The RatButton software was developed with X-Code in object-oriented programming language Objective-C. Stimulus color (sRGB IEC619662.1: $0,240,100$ - a green square) and luminance $\left(150 \mathrm{~cd} / \mathrm{m}^{2}\right.$ at $20 \mathrm{~cm}$ distance) were adapted to rats' vision with the help of a colorimeter (Minolta Chroma Meter xy-1, Minolta Camera) and a photometer (Sekonic DualMaster L-558/L; Sekonic; Jacobs et al., 2001).

Electrical stimulation of the PrL cortex. To determine whether the PrL area-from which the selected $\theta / \beta-\gamma$ transition pattern was recordedwas related to cognitive processes involved in the operant conditioning paradigm, we checked the effects of its electrical stimulation on the ongoing behavioral sequence of touching the iPad and going to collect the rewarded pellet. The PrL cortex was stimulated at the precise moment of each correct touch on the screen.

Histology. At the end of the experiments, rats were deeply reanesthetized with a mixture of ketamine $(100 \mathrm{mg} / \mathrm{kg})$ and medetomidine $(0.1$ $\mathrm{mg} / \mathrm{kg}$ ) and perfused transcardially with saline and $4 \%$ paraformaldehyde in PBS (0.1 M, pH 7.4). Brains were cryoprotected with $30 \%$ sucrose in $\mathrm{PB}$, and coronal sections $(50 \mu \mathrm{m})$ were obtained with a sliding freezing microtome (Leica, SM2000R) and stored at $-20^{\circ} \mathrm{C}$ in $30 \%$ glycerol and $30 \%$ ethylene glycol in PB until used. Selected sections, including the implanted sites, were mounted on gelatinized glass slides and stained using the Nissl technique with $0.1 \%$ toluidine blue to determine the location of stimulating and recording electrodes (Fig. 1C).

Data collection and analysis. Recordings of LFPs, detected $\theta / \beta-\gamma$ transition patterns, $5 \mathrm{~V}$ rectangular pulses corresponding to lever presses, iPad touches, pellet deliveries, and moving images were stored digitally on a computer through an analog-to-digital converter (CED 1401 Plus, Cambridge Electronics Design). Collected data were sampled at $25 \mathrm{kHz}$ for unitary recordings or at $20 \mathrm{kHz}$ for LFPs, with an amplitude resolution of 16 bits. Data were analyzed off-line with the Spike2 software 
A

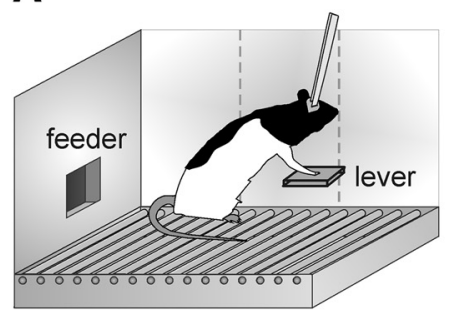

Lever / feeder
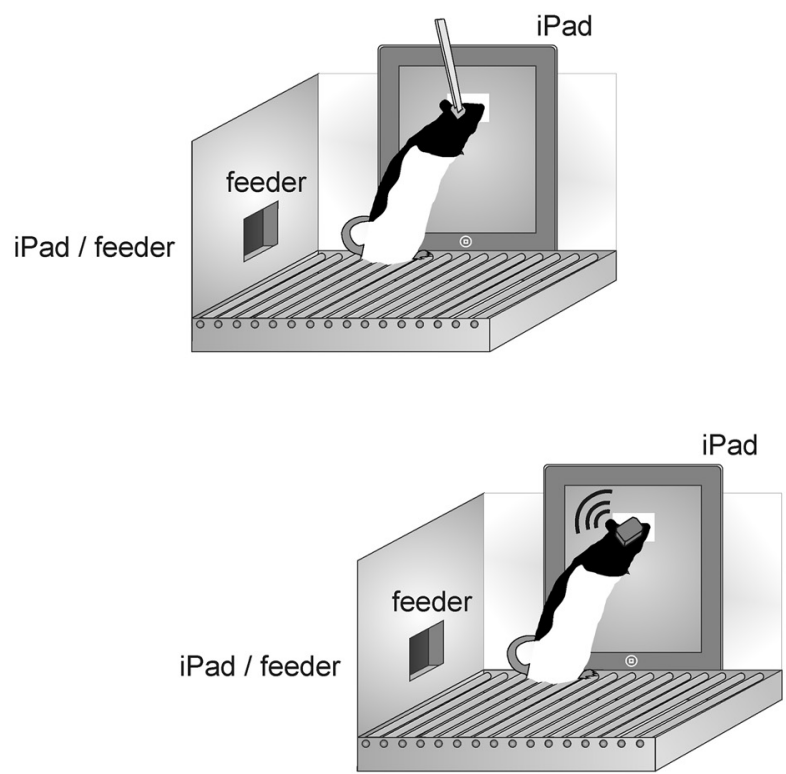

RF1:1
B

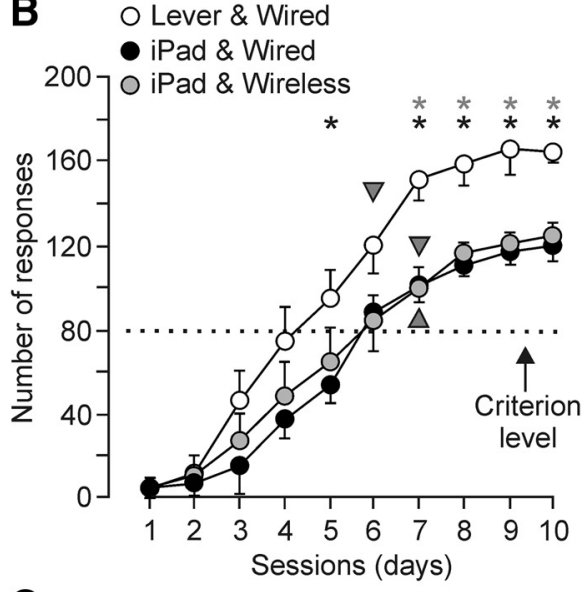

C

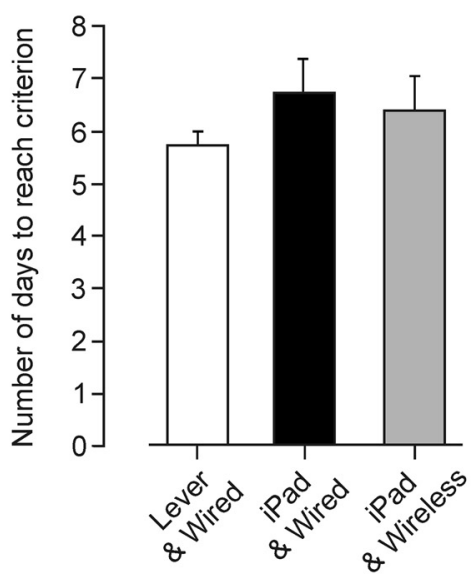

Figure 2. Validation of the touch-screen system compared with a classic lever one using the same instrumental conditioning paradigm. $A$, A group $(n=5)$ of rats were trained in a Skinner box to press a lever to obtain a pellet of food at a fixed 1:1 ratio while the other two groups (wire and wireless; $n=5$ each) were trained in a modified Skinner box to touch a square virtual button displayed on an iPad screen to obtain a pellet at the same ratio. Rats were trained for 10 successive sessions of $20 \mathrm{~min}$ each. $B$, Learning curves corresponding to the three groups of rats. The selected criterion was to obtain $>80$ pellets for two successive sessions (gray triangles). Asterisks indicate significant differences $\left(F_{(2,28,147)}=5.496, p=0.028,2\right.$-way ANOVA) between the group trained in the conventional Skinner box (Lever \& Wired) and the other two (iPad \& Wired and iPad \& Wireless) groups. C, The two touch-screen groups reached the selected criterion after the lever group, but without significant differences $\left(F_{(2,28,12)}=0.634, p=0.547,1\right.$-way ANOVA).

(Cambridge Electronics Design, RRID:SCR_000903) for quantification of animal performance in the Skinner box, $\theta / \beta-\gamma$ pattern presentations and characteristics, power ratio indices of the $\theta / \beta-\gamma$ pattern, and power spectra of selected LFP recordings. Unitary activity was identified and computed with the help of specific Spike2 programs. These computed results were processed for statistical analysis using Sigmaplot 10 (Systat Software, RRID:SCR_003210). Unless otherwise indicated, data are always represented as the mean \pm SEM.

Acquired data were analyzed using one-way or two-way repeatedmeasures ANOVA. The Friedman repeated-measures ANOVA on ranks (nonparametric) statistical test was used if normality or equal variance tests failed. All of them were followed by Holm-Sidak or Tukey's post hoc testing depending on the previous statistical test. The Student's $t$ test was applied when necessary. The corresponding degrees of freedom were reported accompanying the $F, \chi^{2}$, or $t$-statistic values. The analytical procedures of the LFP recordings to obtain the time-frequency representation (spectrogram) and the coherence between pairs of different brain regions (coherograms) were developed with the help of the Chronux toolbox (Matlab, MathWorks, RRID:SCR_005547). In most representations, we selected the following frequency bands: $\theta, 3-12 \mathrm{~Hz} ; \beta, 12-30$ $\mathrm{Hz}$; low $\gamma, 30-50 \mathrm{~Hz}$, and high $\gamma, 50-150 \mathrm{~Hz}$. Comparisons between spectrograms of two different groups of data (Fig. $3 E, F$ ) were processed for statistical analysis using the EEGLAB Statistics toolbox (Matlab, MathWorks, RRID:SCR_010501) and Sigmaplot 10 (Systat Software, RRID:SCR_003210).

\section{Results}

How the $\boldsymbol{\theta} / \boldsymbol{\beta}-\boldsymbol{\gamma}$ transition pattern was selected

Animals were implanted with recording electrodes in PrL and M1 cortices, the hippocampal CA1 area, the mediodorsal thalamic nucleus, nucleus accumbens septi, and the ventral tegmental area (Fig. $1 A-C)$. In a preliminary series of experiments, animals were divided in three groups ( $n=5$ each) and trained until reaching criterion (obtaining $>80$ pellets for two consecutive sessions; see Materials and Methods) in a conventional Skinner box, and in a modified box including a multitouch (iPad) screen, using in both cases a fixed 1:1 rewarding ratio. Animals in the conventional Skinner box were equipped with a wired recording system, while animals belonging to the groups located in the modified Skinner box were implanted with either recording wires or a wireless system (Fig. 2). Although performance with the touch screen was significantly lower than with the lever $\left(F_{(2,28,147)}=5.496, p=0.028\right.$, two-way ANOVA), the three groups of animals reached the selected criterion at similar times $\left(F_{(2,28,12)}=0.634, p=0.547\right.$, one-way ANOVA $)$.

The analysis of LFPs recorded during those operant conditioning sessions, performed in the box equipped with the touch screen, showed the presence of short time periods $(<1 \mathrm{~s})$ characterized by a marked decrease in spectral power, mostly in the $\theta$ 

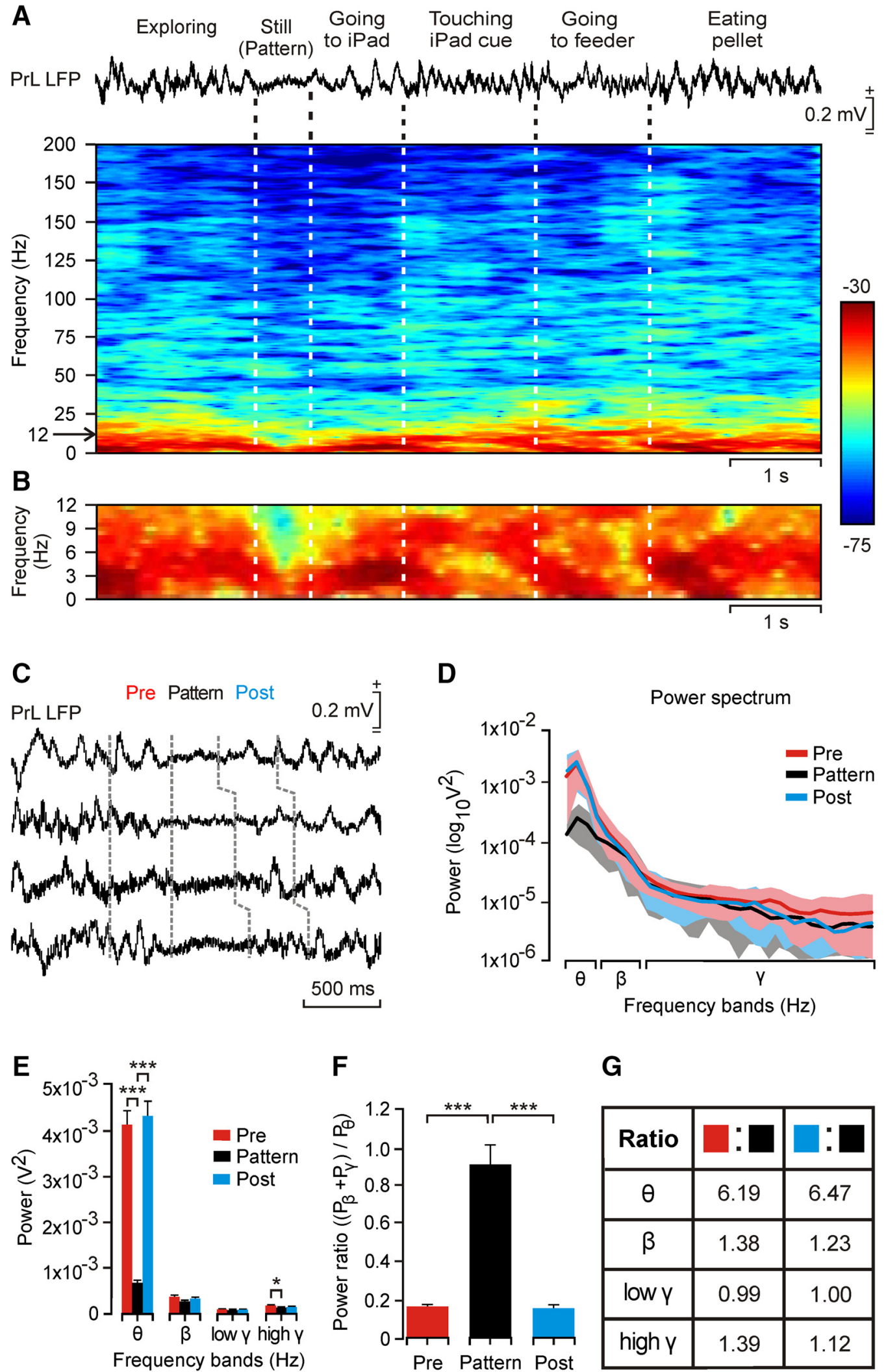

$\mathbf{F}$

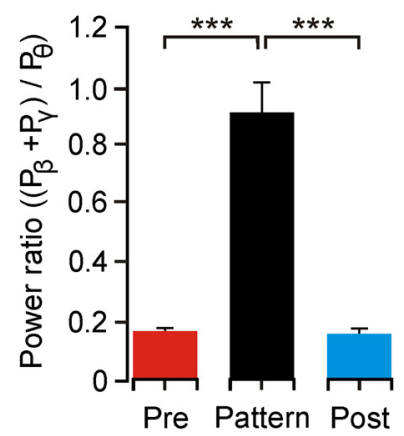

G

\begin{tabular}{|c|c|c|}
\hline Ratio & $: \mathbf{D}$ & $: \mathbf{}$ \\
\hline$\theta$ & 6.19 & 6.47 \\
\hline$\beta$ & 1.38 & 1.23 \\
\hline low $\mathrm{Y}$ & 0.99 & 1.00 \\
\hline high $\mathrm{Y}$ & 1.39 & 1.12 \\
\hline
\end{tabular}

Figure 3. Dynamic changes in LFP activity recorded in the PrL cortex during a sequence of behaviors performed during operant conditioning tasks. $A$, Functional characteristics of the selected $\theta / \beta$ - $\gamma$ transition pattern during a single operant conditioning sequence. From top to bottom are illustrated the behaviors and LFPs recorded in the PrL cortex during this time window. The time-frequency representation of this LFP activity is included below. Note the dominant spectral power (in $\mathrm{dB}$; see the calibration bar) in the $\theta$ band across the whole behavioral sequence, but its decrease during the moment $(>1 \mathrm{~s}$ ) when the rat was still (motionless) before approaching the touch screen. $\boldsymbol{B}$, An enlargement of the time-frequency display corresponding to the band of 0 - 12 $\mathrm{Hz}$. C, Four examples of the selected $\theta / \beta-\gamma$ transition pattern. Pre and Post indicate the LFP activity during the $350 \mathrm{~ms}$ preceding and following the decrease in spectral power in the $\theta$ band. $D$, Averaged ( $n=40$ samples; mean \pm SD) power spectra of each of the three periods (Pre, Pattern, Post) delimited in $C$. Note the large difference in the of power for the $\theta$ band during the pattern. $\boldsymbol{E}$, Analysis of the spectral power of the $\theta, \beta$, low- $\gamma$, and high- $\gamma$ bands for data illustrated in $\boldsymbol{D}\left(\chi^{2}=150.32\right.$; degrees of freedom, $2 ; p \leq 0.001$ for the $\theta$ band; $\chi^{2}=18.98$; (Figure legend continues.) 
A

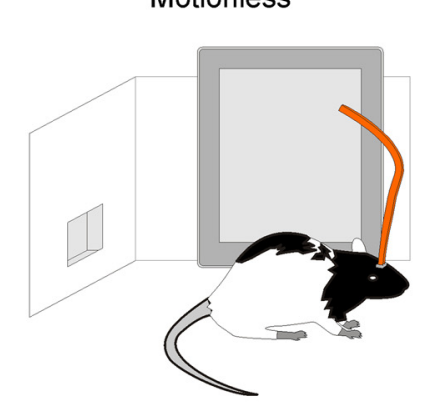

PrL LFP
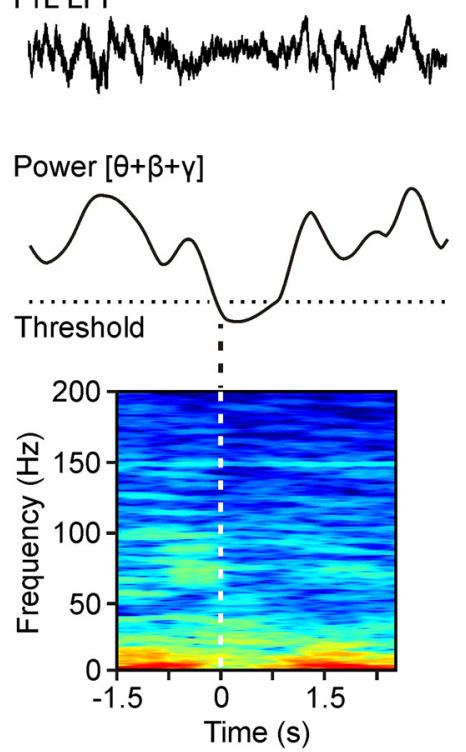

B

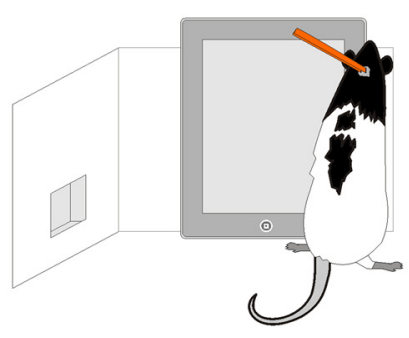

Rearing
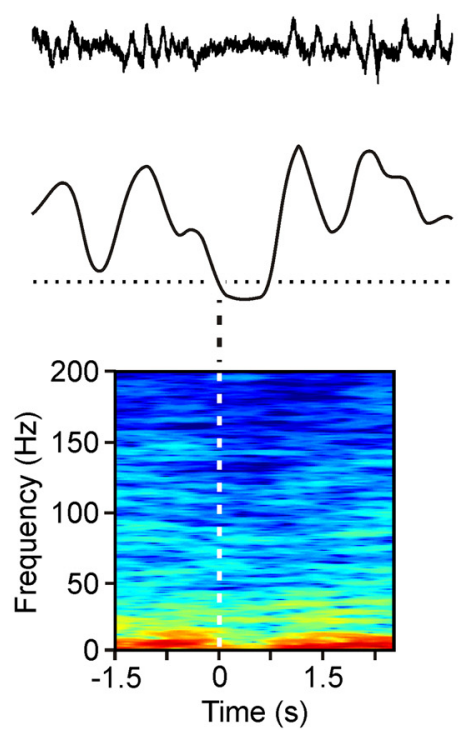

C

Looking into the feeder

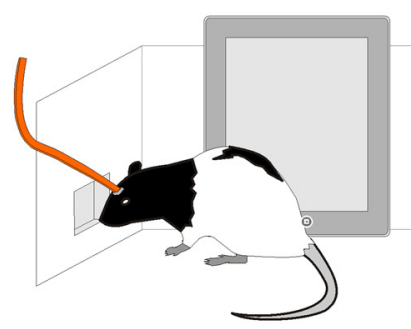

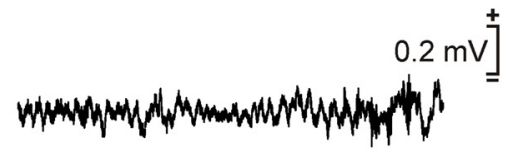

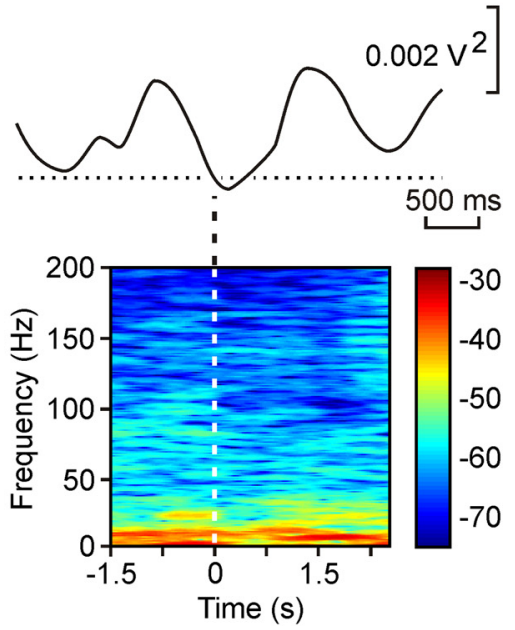

Figure 4. Different behaviors during which the selected $\theta / \beta-\gamma$ transition pattern was recorded in the PrL cortex. $A-C$, Representative examples of LPFs recorded in the PrL cortex during the performance of the illustrated behaviors: $\boldsymbol{A}$, motionless; $\boldsymbol{B}$, rearing; $\boldsymbol{C}$, looking into the feeder. For $\boldsymbol{A}-\boldsymbol{C}$ and from top to bottom are illustrated the selected behavior, the corresponding LFP pattern, the evolution of the added spectral power for the $\theta+\beta+\gamma$ bands across the recorded LFP, and the time-frequency representation of the LFP illustrated above. Note the decreased power of the $\theta$ band during the pattern. Analyses illustrated here were performed with the help of the Chronux application programmed in Matlab.

band (Fig. $3 A-D)$. This peculiar pattern appeared during performance of specific behaviors, such as remaining still (i.e., motionless) in the cage, and during rearing or looking into the feeder (Fig. $4)$. The power in the $\theta$ band was decreased significantly $\left(\chi^{2}=\right.$ 150.32; degrees of freedom, $2 ; p \leq 0.001$, Friedman test) from that present during the periods of time immediately before (Pre) and after (Post; Fig. 3D,E). Smaller but significant differences $\left(\chi^{2}=\right.$ 18.98; degrees of freedom, $2 ; p \leq 0.05$, Friedman test) were found for the high- $\gamma$ band between Pre and Pattern periods (Fig. $3 E$ ).

At this stage, we wondered whether this peculiar $\theta / \beta-\gamma$ transition pattern could be used to trigger the presentation of the selected visual display on the touch screen. In this way, the experimental animal would be able to control the iPad with a specific LFP pattern generated in its PrL cortex. To facilitate this task, we quantified two parameters: the spectral power for the $\theta+\beta+\gamma$ bands (Fig. 5A) and the power ratio $\left[\left(\mathrm{P}_{\beta}+\mathrm{P}_{\gamma}\right) / \mathrm{P}_{\theta}\right]$ during the Pre, Pattern, and Post periods (Fig. $3 F, G$ ), but only the first

\section{$\leftarrow$}

(Figure legend continued.) degrees of freedom, $2 ; p \leq 0.05$ for the high- $\gamma$ band, Friedman test). $\boldsymbol{F}$, Power ratio of $\beta+\gamma$ bands with respect to the $\theta$ band [power ratio: $\left.\left(\mathrm{P}_{\beta}+\mathrm{P}_{\gamma}\right) / \mathrm{P}_{\theta}\right]$ for each of the three regions ( $n=100$ samples collected from 5 animals; $\chi^{2}=136.82$; degrees of freedom, $2 ; p \leq$ 0.001 , Friedman test). $G$, Ratio between the spectral powers of each frequency band corresponding to LFPs recorded before and during the pattern (Pattern/Pre, second column) and after and during the pattern (Pattern/Post, third column). These ratios were computed for data illustrated in $\boldsymbol{E}$. parameter was used as an iPad trigger. Pre and Post periods presented similar power ratio values $(0.17$ and 0.16 , respectively; $\chi^{2}=136.82$; degrees of freedom, $2 ; p>0.05$; Tukey's post hoc test for Friedman test). In contrast, the power ratio for the Pattern period reached a value significantly larger $\left(0.91 ; \chi^{2}=136.82\right.$; degrees of freedom, $2 ; p \leq 0.001$, Tukey's post hoc test for Friedman test). Quotients between Pre/Pattern and Post/Pattern periods for each of the mentioned $(\theta, \beta$, and $\gamma)$ power spectral bands yielded similar values (Fig. $3 G$ ). The quotient for the $\theta$ band was $>6$ times larger for the Pre/Pattern (6.19) and Post/Pattern (6.47) periods, but the other frequency bands presented minor differences (Fig. $3 G$ ). On the whole, these analyses indicate that the selected $\theta / \beta-\gamma$ transition pattern was mainly characterized by a notable decrease in the $\theta$ band during the Pattern period.

Use of the selected $\theta / \beta$ - $\gamma$ transition pattern to trigger the appearance of a visual display on the touch screen

In a second series of experiments, a group of implanted rats were trained to touch a visual display that appeared automatically on the touch screen (Automatic cue; Fig. $5 B-D$ ). In this situation, they reached criterion between the fifth and the ninth conditioning sessions. Rats were maintained with this operant task for $\leq 11$ sessions and then they were trained to activate the appearance of the same visual display with the generation of the selected $\theta / \beta-\gamma$ transition pattern (Pattern-evoked cue; Fig. 5B-D). To trigger 


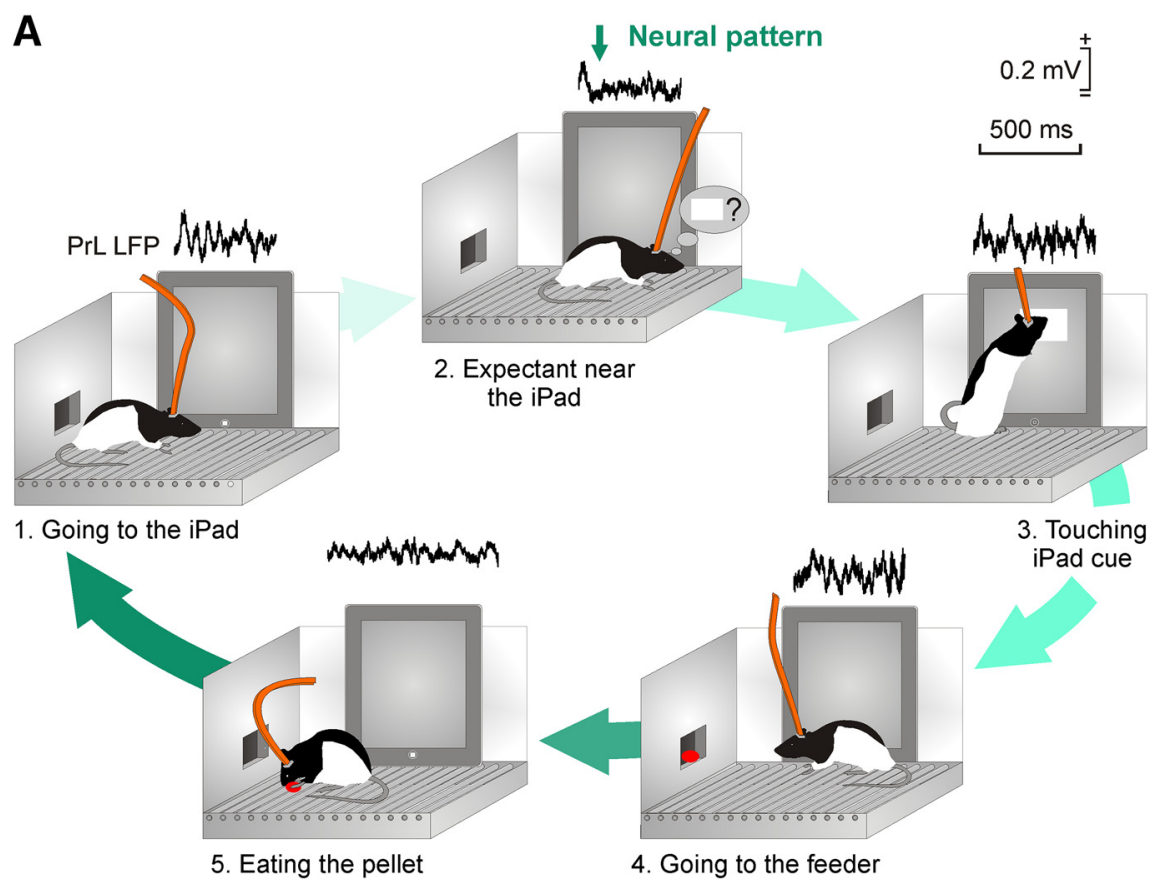

B
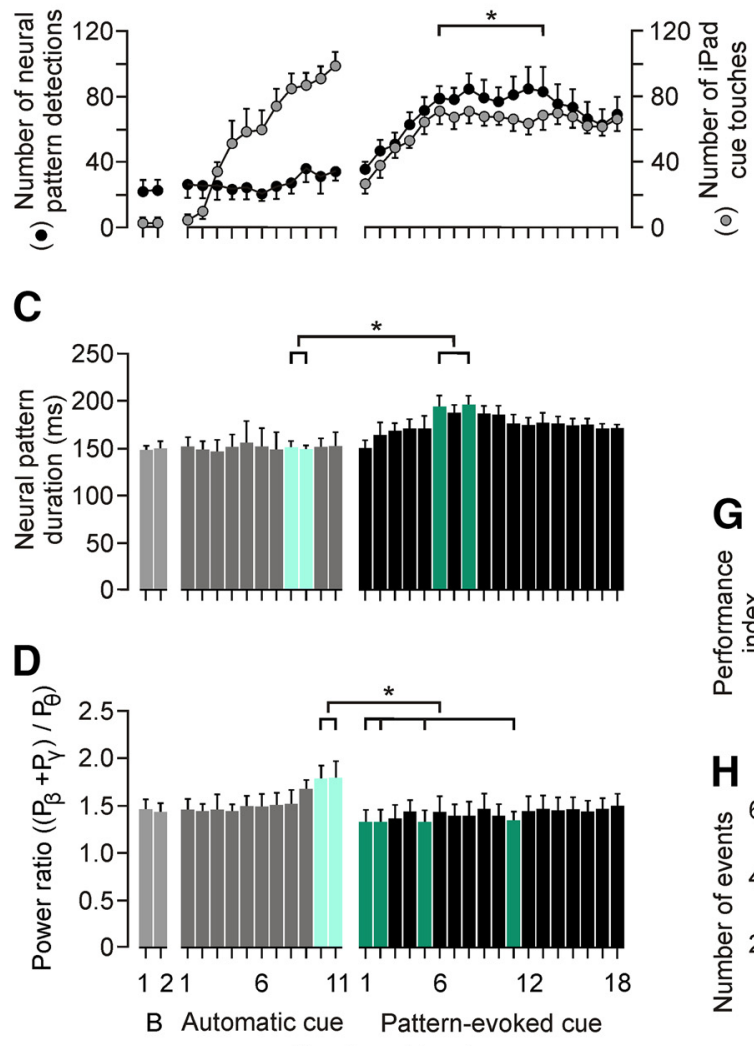

Sessions (days)
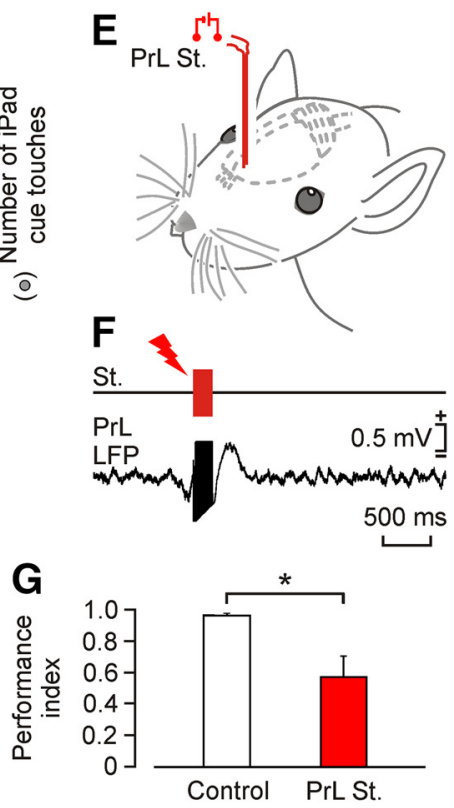

H

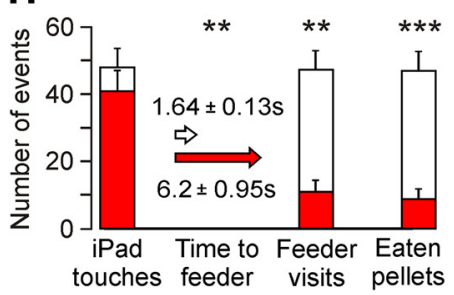

Figure 5. Evolution of the selected $\theta / \beta-\gamma$ transition pattern across training with the touch-screen task. $\boldsymbol{A}, \mathrm{A}$ diagram of behaviors performed by the experimental animal during a complete sequence of operant conditioning with the touch screen. The corresponding LFP evoked in the PrL cortex during the corresponding behavior is illustrated above each sketch. $\boldsymbol{B}$, Evolution of the number of screen touches during baseline recordings, when the square virtual button display appeared spontaneously on the touch screen (Automatic cue) and when its appearance was triggered by the $\theta / \beta-\gamma$ transition pattern recorded in the PrL cortex (Pattern-evoked cue). Data collected and averaged from 10 animals. Note that pattern presentation showed no significant $(p>$ $0.05)$ changes during automatic cue sessions, but increased significantly $\left(F_{(1,9,339)}=30.096, p \leq 0.001,2\right.$-way ANOVA) for pattern-evoked cue sessions. $\boldsymbol{C}$, Evolution of pattern duration across training sessions illustrated in $\boldsymbol{B}$. Note the slight increase in the appearance of a visual display on the touch screen, a program was implemented to detect the Pre-to-Pattern transition with a delay of $<0.25 \mathrm{~s}$ (Fig. $5 A$ ). In this situation, rats presented a constant increment in the number of $\theta / \beta-\gamma$ transition patterns during the first eight sessions, followed by a stabilization $(\approx 80$ patterns and $\approx 60$ touches) from the ninth to the 18th training sessions. Although the number of evoked patterns reached criterion, the number of screen touches did not. This lower performance was not due to the presence of recording wires, since wireless recordings performed in an additional group of rats produced similar results (Fig. $2 B, C$ ).

As illustrated in Figure $5 B$, there were no significant changes in the number of $\theta / \beta$ - $\gamma$ transition patterns for the two baseline and 11 training sessions in which the visual display was presented automatically $(p>0.05)$. In contrast, during the sessions in which the visual display was triggered by the $\theta / \beta$ - $\gamma$ transition pattern, the number of screen touches progressed in parallel with a significant $\left(F_{(30,270,310)}=9.007, p \leq 0.001\right.$, one-way ANOVA) increase in the number of pattern detections from the sixth to the 13th conditioning sessions.

We checked whether the use of the $\theta / \beta-\gamma$ transition pattern to trigger the iPad modified its properties regarding duration and the values reached by the power ratio index. As shown in Figure $5 C$, when used to trigger the iPad (Pattern-

$\leftarrow$

duration across the first sessions in which the pattern was used to trigger the visual display on the touch screen. Significant differences are indicated ( $\chi^{2}=47.098$; degrees of freedom, $30 ; p \leq 0.05$, Friedman test). This increase in pattern duration disappeared after the first 10 pattern-evoked cue sessions. $\boldsymbol{D}$, Evolution of the power ratio $\left[\left(\mathrm{P}_{\beta}+\mathrm{P}_{\gamma}\right) / \mathrm{P}_{\theta}\right]$ across training sessions. Power ratio was larger for automatic than for pattern-evoked cue sessions. Significant differences are indicated $\left(\chi^{2}=55.423\right.$; degrees of freedom, 30; $p \leq 0.05$, Friedman test). $\boldsymbol{E}, \boldsymbol{F}$, Experimental design for animal stimulation in the PrL cortex. The experimental animal was stimulated for $200 \mathrm{~ms}(50 \mu \mathrm{s}$ pulses at $200 \mathrm{~Hz}$ ) the moment it touched the screen. A total of five rats/group was used. G, Differences in the performance index [index: $\left(N_{\text {feeder }}+N_{\text {eat }}\right) /\left(N_{\text {feeder }}+\right.$ $\left.N_{\text {touches }}\right)$, where $N=$ number of events]. Control rats reached an index of $0.97 \pm 0.01$ (white bar), while stimulated rats reached significantly lower values $\left(0.58 \pm 0.15, t_{(8,0.05)}=\right.$ $2.586, p \leq 0.05$, Student's $t$ test). $\boldsymbol{H}, 0$ ther quantitative differences in operant conditioning performance between the control and the stimulated groups. Although the number of screen touches was similar for the two groups $\left(t_{(8,0.05)}=\right.$ $1.043 ; p=0.328$; Student'st test), the stimulated group spent a significantly longer time reaching the feeder $(p=0.001$; Mann-Whitney rank sum test), paid fewer visits to the feeder $\left(t_{(8,0.05)}=5.008, p<0.001\right.$, Student's $t$ test $)$, and ate fewer pellets $\left(t_{(8,0.05)}=5.334, p<0.001\right.$, Student's $t$ test $)$. 
evoked cue), the duration of the pattern increased steadily from the first to the eighth training sessions (from 150 to 197 $\mathrm{ms} ; \chi^{2}=47.098$; degrees of freedom, 30; $p \leq 0.05$, Friedman test), decreasing afterward to intermediate values (from 186 to $160 \mathrm{~ms}$ ). In contrast, the power ratio reached peak values at the end of the automatic cue sessions, being significantly smaller during pattern-evoked cue sessions $\left(\chi^{2}=55.423\right.$; degrees of freedom, 30; $p \leq 0.05$; Friedman test; Fig. $5 D)$. Thus, the use of the $\theta / \beta-\gamma$ transition pattern to trigger the touch screen introduces small but significant changes in its duration and spectral power characteristics.

\section{The electrical stimulation of the PrL cortex prevented the proper performance of the operant conditioning task}

It is well known that the PrL cortex from which the $\theta / \beta$ - $\gamma$ transition pattern was recorded is involved in cognitive, volitional, and valuation processes (Fuster, 2015; Kable and Glimcher, 2009; Graybeal et al., 2011). Specifically, its electrical stimulation seems to prevent the proper performance of an operant conditioning task (Jurado-Parras et al., 2012). In this regard, we checked whether the recorded area was also involved in these volitional processes. For this, we stimulated (a train of $200 \mathrm{~Hz}$ for $200 \mathrm{~ms}$ ) the PrL cortex in animals trained to touch the visual display to obtain a pellet as a reward (Fig. $5 E-H$ ).

Control rats performed the task consistently, visiting the feeder and getting the pellet after each screen touch, with a performance index close to $1(0.97 \pm 0.01$; Fig. $5 G)$. In contrast, electrical stimulation of the PrL cortex (Fig. 5F) disturbed this sequential behavior because, although cortically stimulated rats touched the screen as many times as controls $\left(t_{(8,0.05)}=1.043, p=0.328\right.$, Student's $t$ test), they either did not visit the feeder $\left(t_{(8,0.05)}=5.008, p=\right.$ 0.001 , Student's $t$ test) or they tried to visit it but returned to the touch screen without eating the reward $\left(t_{(8,0.05)}=5.334, p \leq\right.$ 0.001 , Student's $t$ test). In addition, the time elapsed before visiting the feeder was significantly increased $\left(U_{(8,0.05)}<0.001\right.$, $p=0.008$, Mann-Whitney rank sum test; Fig. $5 H)$. Rats with stimulated PrL cortices produced a significantly $\left(t_{(8,0.05)}=2.586\right.$, $p=0.032$, Student's $t$ test) lower performance index $(0.58 \pm$ 0.15 ) than rats in the control group (Fig. $5 G$ ), indicating that the stimulated rats did not visit the feeder and get the pellets delivered as effectively as controls did (Fig. $5 H$ ). These results suggest that the electrical stimulation of PrL recording sites disturb the proper performance of specific behavioral sequences related to the operant task.

Unitary activity in the PrL cortex in presence of the $\theta / \beta-\gamma$ transition pattern

Particular attention was paid to the firing activities of PrL cortex neurons recorded simultaneously with the spontaneous appearance of the selected $\theta / \beta-\gamma$ transition pattern (Automatic cue; Fig.
B Pattern-evoked cue
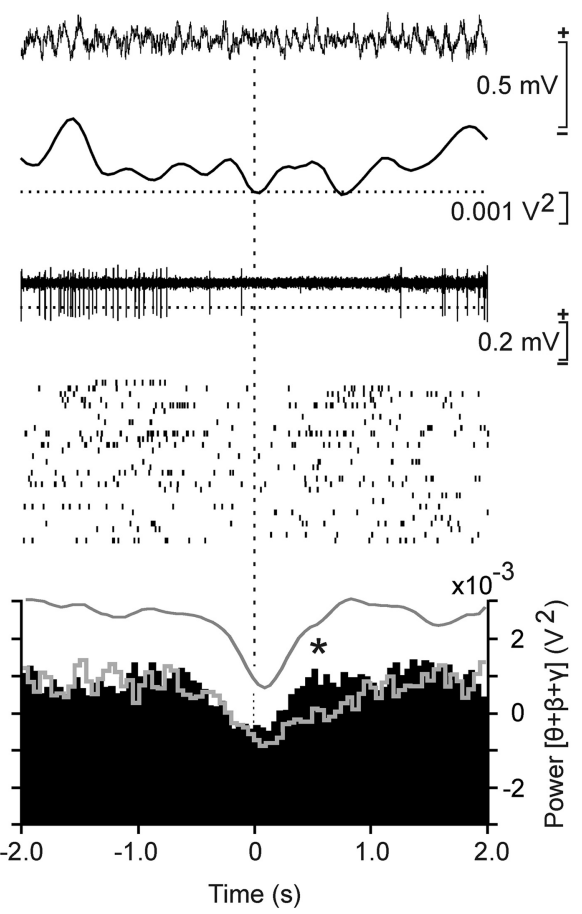

Time (s)

Figure 6. Unitary activity in the PrL cortex when the selected $\theta / \beta$ - $\gamma$ transition pattern was generated spontaneously and when used to trigger touch-screen cues. $\boldsymbol{A}$, Unitary activity recorded in the PrL cortex coincided with the spontaneous appearance of the $\theta / \beta$ - $\gamma$ spectral power for the $\theta+\beta+\gamma$ bands across the recorded LFP, the firing activity of a PrL neuron, the raster plot of $\geq 25$ successive trials period. The statistical analysis of collected data indicated that there was a depression in the firing rate of the recorded neurons $(n=36)$ during the transition pattern $\left(F_{(78,320,399)}=6.994, p<0.05\right.$, 1-way ANOVA) and that this depression was recovered significantly $\left({ }^{*} t_{(19,0.05)}=-6.552, p<0.001\right.$, paired Student's $t$ test $)$ before, by the same neuronal group, during Pattern-evoked-cue trials.

$6 A)$ or during its use to trigger the visual display in the iPad (Pattern-evoked cue; Fig. 6B). Most (80\%) of the recorded units (36 of 45 ) were considered as putative PrL pyramidal neurons, based on the duration of the recorded spikes $(>0.5 \mathrm{~ms})$ and on their spontaneous mean firing rates (2-15 spikes/s). These PrL neurons presented sustained tonic firings interrupted sometimes by short bursts of activity. Because of their firing properties, they appeared to be similar to the regular-spiking, slow-adapting pyramidal cells recorded by Dégenètais et al. (2002) in anesthetized rats and by Leal-Campanario et al. (2013) in alert behaving rabbits. In accordance with the collected results, averaged PrL pyramidal neurons presented a significant $\left(F_{(78,320,399)}=6.994\right.$, $p<0.05$, one-way ANOVA) decrease in their mean firing rate coinciding with the spontaneous appearance of the $\theta / \beta-\gamma$ transition pattern (Fig. $6 A$ ) or during its use as Pattern-evoked cue (Fig. $6 B)$. Nevertheless, the same set of neurons recovered their firing rates significantly $\left(t_{(19,0.05)}=-6.552, p<0.001\right.$, paired Student's $t$ test) faster following $\theta / \beta-\gamma$ transition patterns used as a cue than during their spontaneous presentations.

In addition, and as illustrated in Figure 7 , the selected $\theta / \beta-\gamma$ transition pattern appeared to coincide with a small $(\approx 50 \%)$ decrease in vibrissal activity, suggesting that this specific activity of the PrL cortex was not related to somatosensory information or exploratory behaviors, contrary to what has been described for the S1 and M1 cortical areas (Zagha et al., 2013). 
A

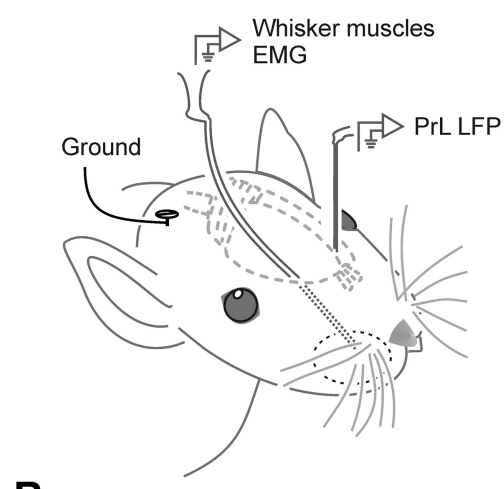

B

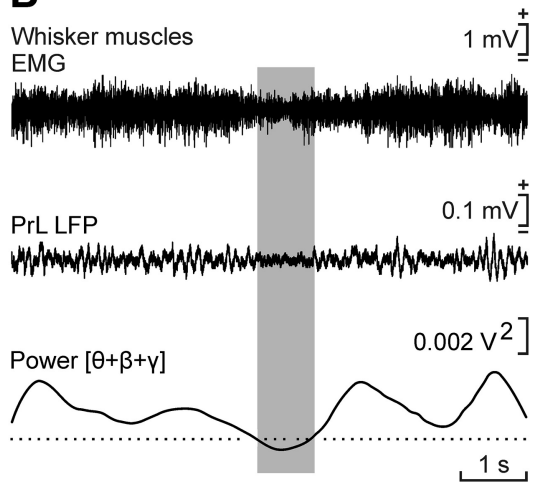

C

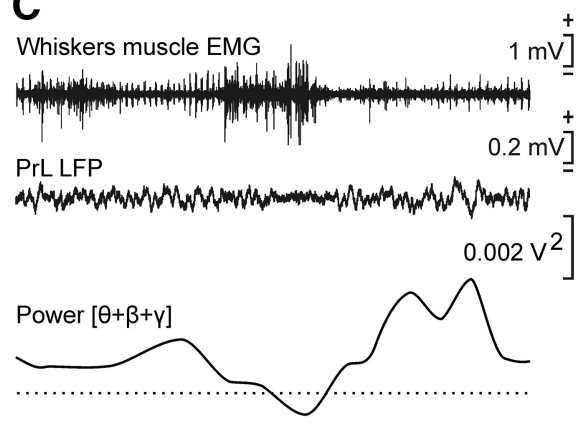

D

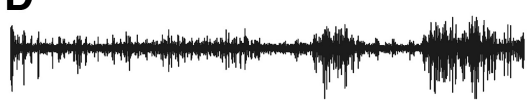

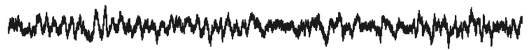

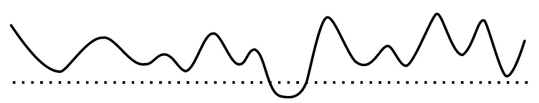

$E$
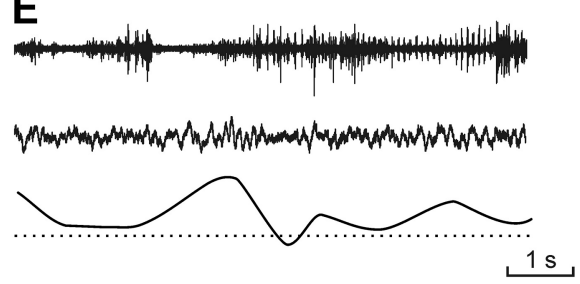

Figure 7. The selected $\theta / \beta-\gamma$ pattern was not related to whisker motor responses. $A$, Experimental design. The electromyographic (EMG) activity of whisker muscles and the LFPs generated in the PrL cortex were recorded simultaneously in alert behaving rats during operant conditioning sessions in which the iPad was triggered by the $\theta / \beta$ - $\gamma$ pattern. $\boldsymbol{B}$, Averaged $(n=175$ traces collected from 3 animals) EMG recordings collected from whisker muscles and LFPs collected from the PrL cortex. The average was triggered when the summed power of $\theta+\beta+\gamma$ bands reached the selected minimum value. Although the averaged EMG activity of whisker muscles presented a minimum value during the presence of the selected pattern, no linear relationship was observed between the two variables $(r=0.073$; degrees of freedom, 348; $p=0.336$, Pearson's $r)$. $\boldsymbol{C}-\boldsymbol{E}$, Three representative examples of LFPs recorded during the presence of the selected pattern in the PrL cortex accompanied by different levels of activity in whisker muscles. These results suggest that the two neural and muscle activities were not correlated.

Effects of the use of the $\theta / \beta$ - $\gamma$ transition pattern as a trigger of the touch screen on the power spectra of LFPs recorded in the PrL cortex

We were interested in determining whether the use of the LFP pattern as a trigger modified its spectral characteristics. As mentioned above, rats already trained to touch the screen to obtain a reward generated spontaneously the $\theta / \beta$ - $\gamma$ transition pattern when looking toward the feeder, remaining motionless in the cage, or rearing (Figs. $4 A-C, 8 A)$. In this situation, the pattern was present $24 \pm 1.5$ times per recording session (Fig. $5 B$ ). When the pattern was used to activate the touch screen, the rat usually generated it by standing still near the iPad (Figs. 4A, 8C). Figure $8 B, D$ shows an averaged (150 times) time-frequency representation $(1250 \mathrm{~ms})$ triggered by the $\theta / \beta-\gamma$ transition pattern during the two different experimental situations illustrated in Figure $8 A, C$. As illustrated in Figure $8 G-I$, a quantitative analysis of collected data indicated that, when the LFP pattern was used as a trigger, it presented a lower power in the $\beta$ band $\left(t_{(298,0.05)}=5.949, p=0.001\right.$, Student's $t$ test $)$ during Pre, Pattern, and Post periods and in the low- $\gamma$ band $\left(t_{(298,0.05)}=7.863, p<0.001\right.$, Student's $t$ test) during the Pattern and Post periods. Finally, there was a decrease $(p \leq 0.05)$ in the high- $\gamma$ band during the Post period. Interestingly, there was no significant change in the $\theta$ band for any of these three periods $\left(t_{(298,0.05)} \geq-1.444, p \geq 0.150\right.$, Student's $t$ test $)$.

A precise study of the high- $\gamma$ band revealed a significant decrease ( $p \leq 0.05)$ of the power in a narrow range of frequencies $(115-122 \mathrm{~Hz}$;
Fig. $8 F$ ) that coincided with the detection of the LFP pattern when it was used to activate the touch-screen cue. Similar results were obtained for the low- $\gamma$ band and, particularly, in the range of frequencies between 32 and $41 \mathrm{~Hz}(p \leq 0.05)$. Interestingly, these frequencies did not change their spectral powers before and after the Pattern period. The opposite alternation in the presence $(p \leq 0.05)$ or absence $(p>0.05)$ of differences between the two experimental phases in the three different time periods (Pre: -250 to $0 \mathrm{~ms}$; Pattern: 0-250 ms; Post: $250-500 \mathrm{~ms}$ ) was observed in some frequencies of the $\theta, \beta$, and low- $\gamma$ bands (Fig. $8 E$ ).

\section{Analysis of coherence between the six recording sites across training} An interesting question was to determine whether the selected $\theta / \beta-\gamma$ transition pattern was originated in a neural area other than the PrL cortex or was propagated from it to other cortical and subcortical areas. In Figure $9 A$ are illustrated the LFPs recorded in the six selected areas during a complete operant conditioning sequence from going to the iPad to touch the visual display until eating the provided pellet. As illustrated in Figure $9 B$, there was a very low coherence for the $\theta$ band $(0.06 \leq C \leq$ $0.19)$ and a slightly higher one for the $\beta$ band $(0.29 \leq C \leq 0.51)$ when the PrL cortex was compared with the other five regions. Only the $\gamma$ band (especially at high frequencies) presented a high coherence $(C \leq 0.62)$ between the PrL and M1 cortices.

A detailed analysis of coherence between these six regions revealed peaks of coherence in some frequencies, with values close to 0.6 during the generation of the $\theta / \beta-\gamma$ transition pattern. When LFPs recorded in the PrL cortex were compared with those recorded in the M1 cortex, three peaks of medium-level coherence were found in low- $\gamma$ and high- $\gamma$ frequencies $(42-65,109-122$, and 139-150 $\mathrm{Hz} ; 0.60 \leq C \leq 0.67$; Fig. $9 C$ ). Coherograms for LFPs recorded from PrL-mediodorsal thalamic nucleus and PrL-ventral tegmental area showed a generalized low coherence in most of the bands, apart from the low- $\gamma$ band (43-63 and 45-51 Hz respectively; $0.60 \leq C \leq 0.67$; Fig. $9 C, D)$, mostly during the Pre and the beginning of the Pattern periods. As illustrated in Figure $4 D$, the nucleus accumbens septi and the hippocampal CA1 area presented the lowest coherence in the $\theta, \beta$, and low- $\gamma$ bands $(0.06 \leq C \leq 0.37)$, even though a narrow range of frequencies (between 146 and 150 $\mathrm{Hz}$ ) reached coherence values from 0.60 to 0.62 for PrL and nucleus accumbens septi LFPs.

\section{Discussion}

Present results indicate that the use of definite LFP patterns generated in the PrL cortex can be safely used as an alternative for functional studies associated with BMIs and other neural processes related to neurofeedback procedures. As already described, and further confirmed here, the PrL cortex is involved in volitional and valuation processes (Vertes, 2004; Hoover and Vertes, 2007; Kable and Glimcher, 2009; Graybeal et al., 2011; Fuster, 
A Spontaneous
PrL pattern

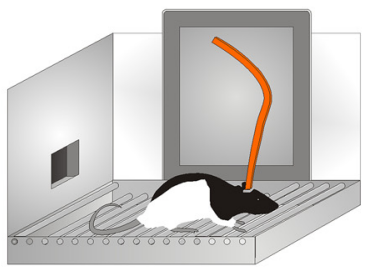

B
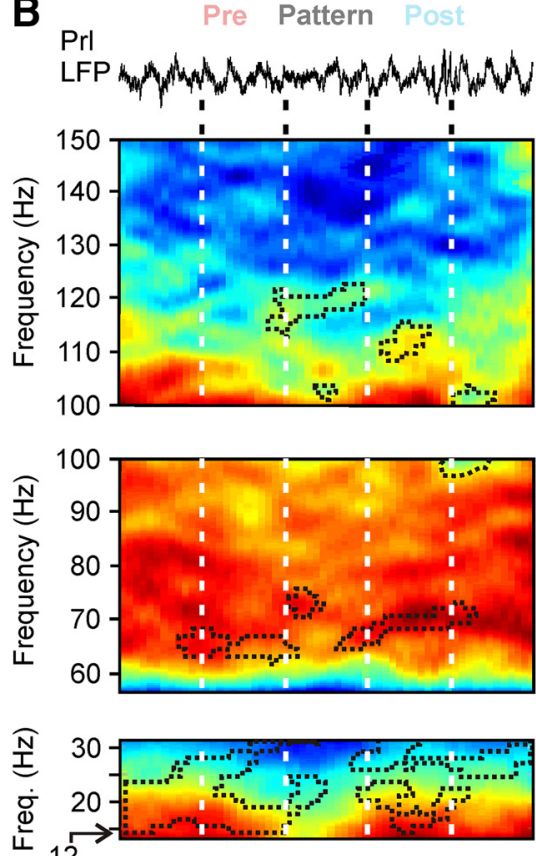
ㄴ. 12

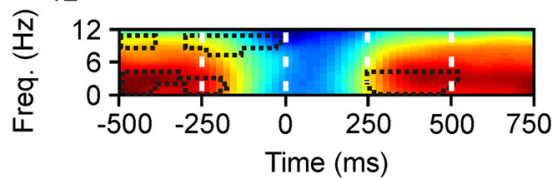

G

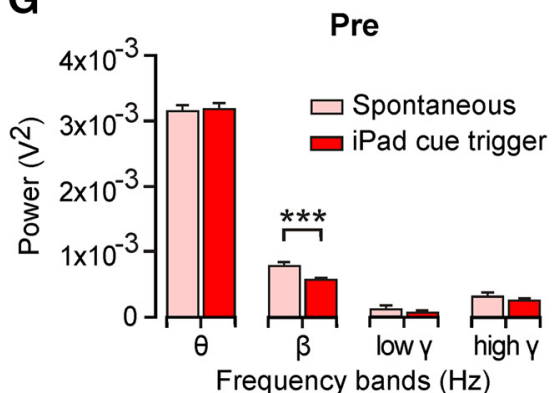

C PrL pattern used as iPad cue trigger

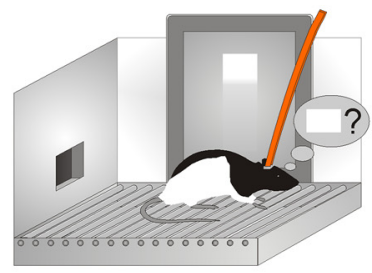

D Pre Pattern Post
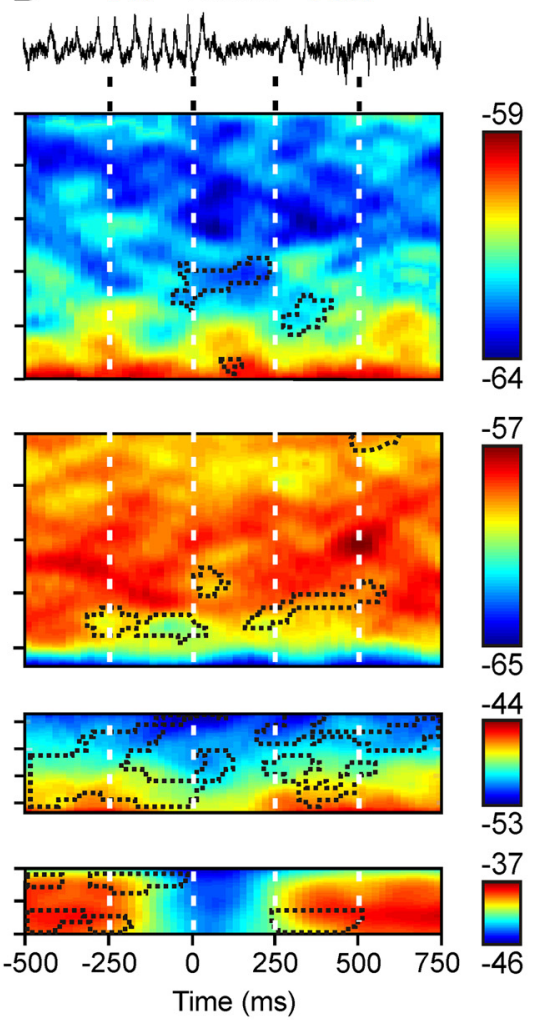

H

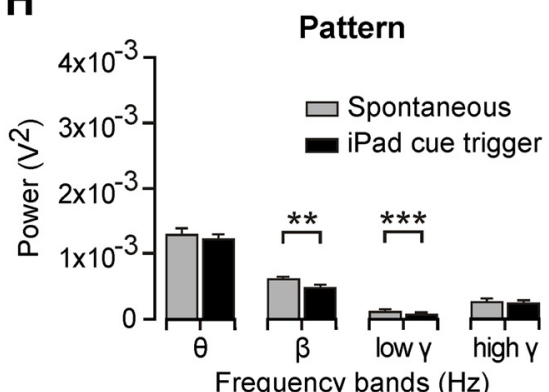

E

Spontaneous $=\mathrm{iPad}$ cue trigger Spontaneous $\neq \mathrm{iPad}$ cue trigger

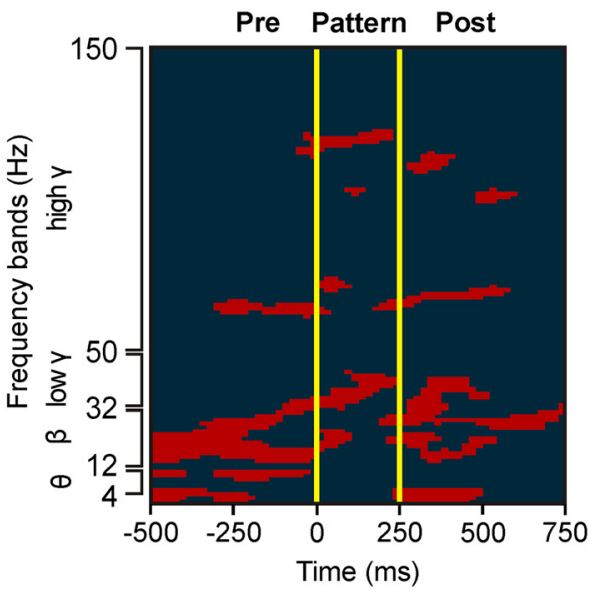

$\mathbf{F}$

$\square$ Spontaneous > iPad cue trigger

Spontaneous $=$ iPad cue trigger

Spontaneous $<$ iPad cue trigger

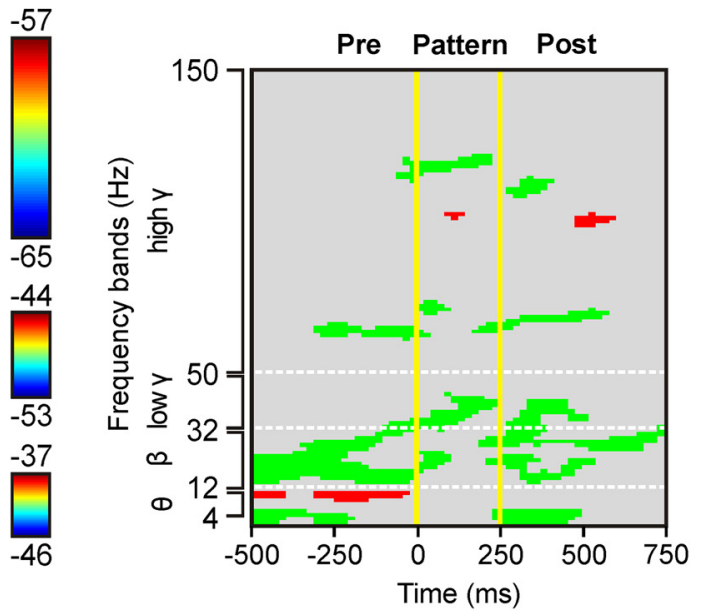

I

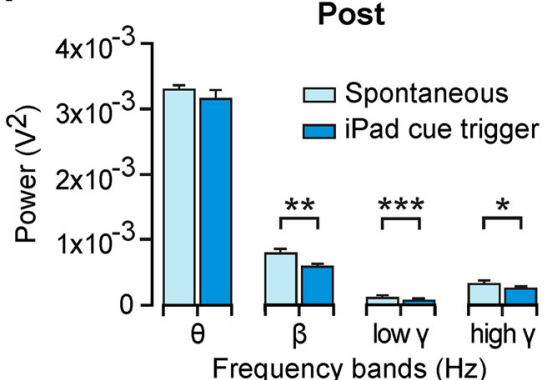

Figure 8. Differences in the spectral properties of the selected $\theta / \beta-\gamma$ transition pattern when generated spontaneously and when used to trigger touch-screen cues. $A$, As illustrated in Figure $5 B-D$, the spontaneous presentation of the $\theta / \beta-\gamma$ transition pattern during the automatic presentation of the visual (square virtual button) cue on the touch screen was recorded. $\boldsymbol{B}$, At the top is illustrated a representative example of LFPs, including the presence of the $\theta / \beta-\gamma$ transition pattern evoked spontaneously. Below are illustrated timefrequency representations averaged from 150 traces $(n=5$ rats), including the selected pattern recorded from automatic cue sessions. $\boldsymbol{C}, \boldsymbol{D}$, The same as for $\boldsymbol{A}$ and $\boldsymbol{B}$, but corresponding to LFPs recorded during pattern-evoked cue operant conditioning sessions ( 150 traces collected from 10 rats). Areas surrounded by dots correspond to observed differences between the two time-frequency displays. $\boldsymbol{E}$, Significant differences $(p \leq 0.05)$ between the averaged time-frequency displays illustrated in $\boldsymbol{B}$ and $\boldsymbol{D}$. $\boldsymbol{F}$, Same as in $\boldsymbol{E}$, but indicating when the spectral power was larger for $\theta / \beta$ - $\gamma$ patterns evoked spontaneously (green areas) versus those cases in which the spectral power was larger for patterns used to trigger the visual cue (red areas). G-I, Spectral analysis for LFPs collected before (Pre, $\boldsymbol{G}$ ), during (Pattern, $\boldsymbol{H}$ ), and after (Post, $\boldsymbol{I}$ ) for data illustrated in $\boldsymbol{B}$ and $\boldsymbol{D}$. Significant differences are indicated. ${ }^{*} p<0.05$; ${ }^{* *} p<0.01$; ${ }^{* * *} p<0.001$, Student's $t$ test. 
A

$$
\begin{array}{cc}
\text { A } & \multicolumn{2}{c}{\text { Touching }} \\
\text { Going } & \text { iPad cue } \\
\text { to iPad } & \text { Still } \downarrow \text { Going } \\
& \text { to feeder }
\end{array}
$$

PrL

M1

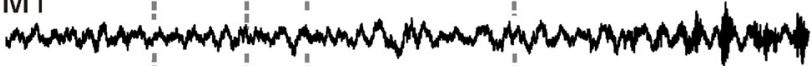

MD

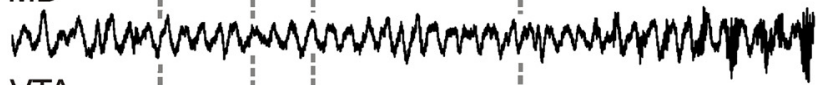

VTA

NAC

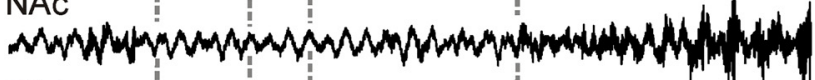

CA1

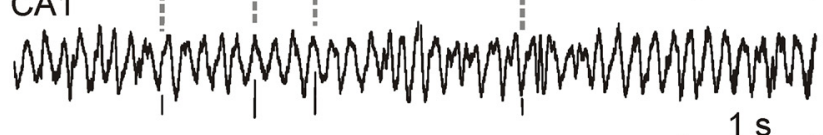

Eating

pellet
B

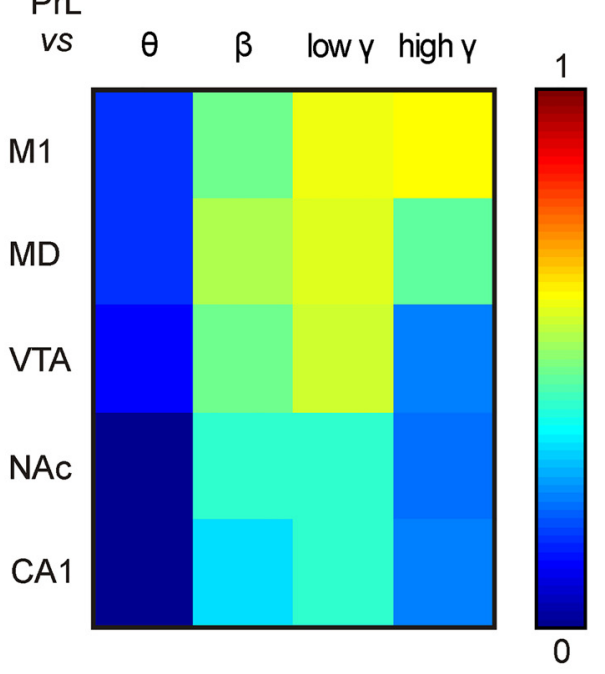

C

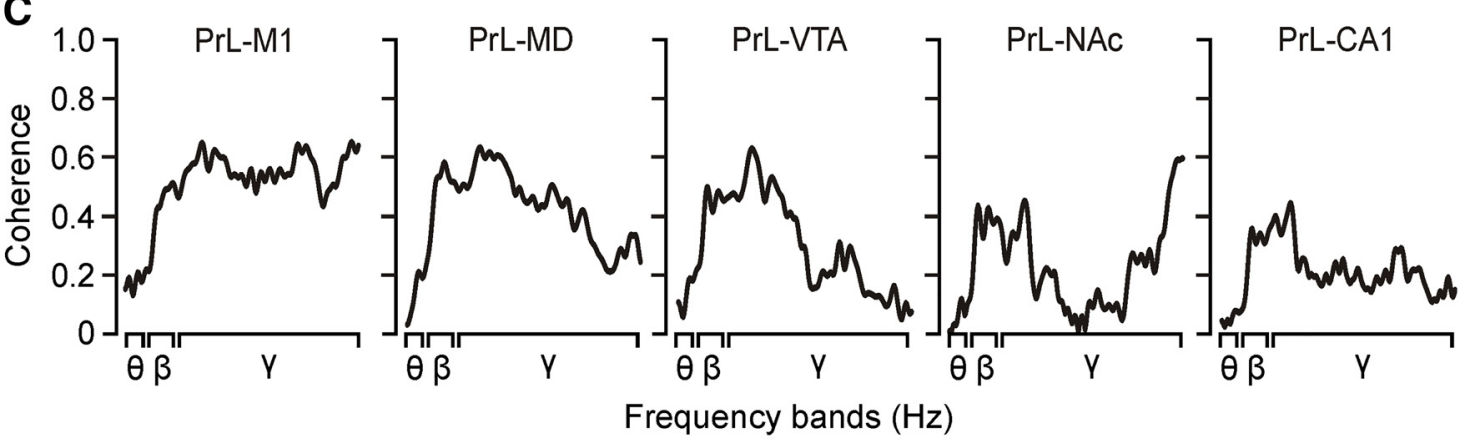

D
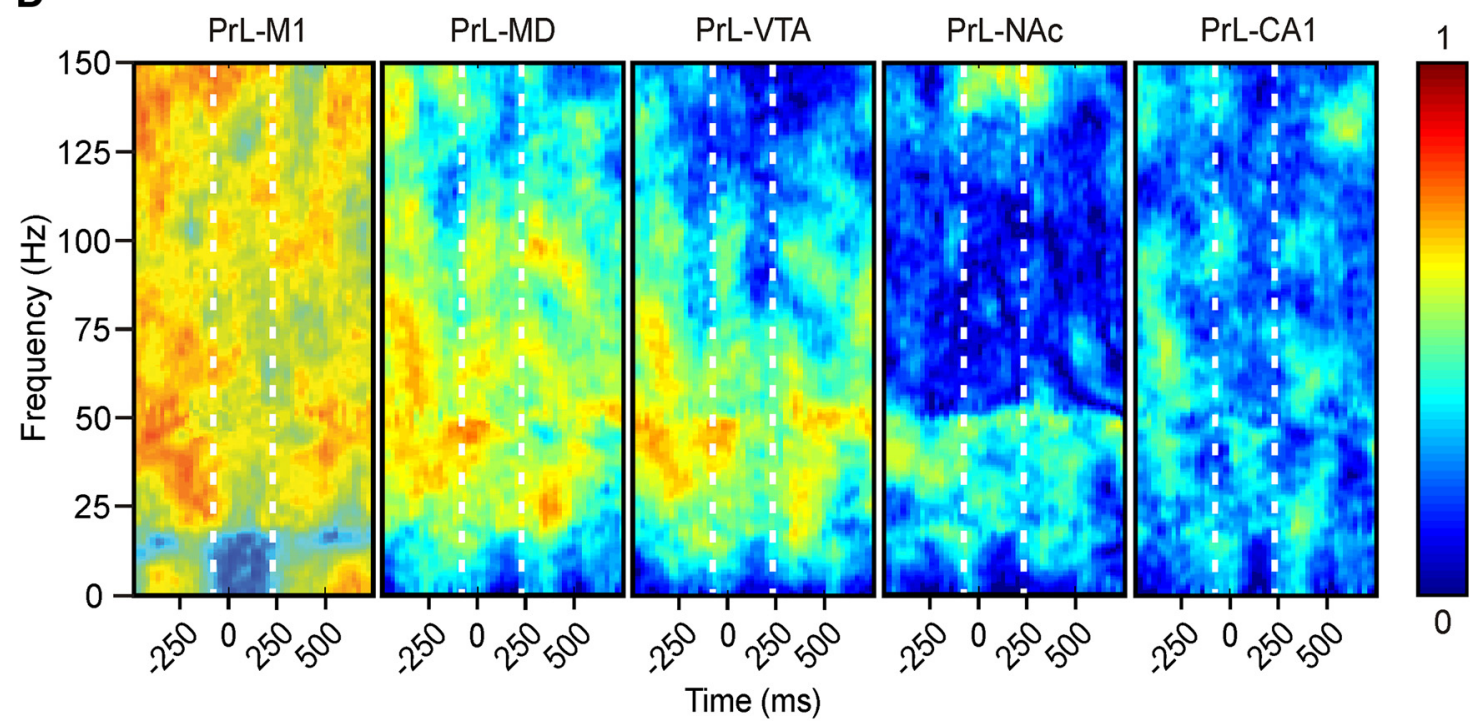

Figure 9. The selected $\theta / \beta$ - $\gamma$ transition pattern is restricted to the PrL cortex. $\boldsymbol{A}$, LFPs recorded simultaneously in six different cortical and subcortical sites during a sequence of the touch-screen operant conditioning task. $\boldsymbol{B}$, Analysis of coherence between the $\theta / \beta$ - $\gamma$ transition patterns recorded in the PrL cortex and the other five recording sites for the $\theta$, $\beta$, low- $\gamma$, and high- $\gamma$ bands. Note that the highest coherence $(0.62$, see color scale) was between the PrL and the M1 area of the motor cortex for the high- $\gamma$ band. $\boldsymbol{C}$, Coherence profiles for the activity of $\theta$, $\beta$, low- $\gamma$, and high- $\gamma$ bands in the PrL cortex and the other five recording sites during the appearance of the $\theta / \beta$ - $\gamma$ transition pattern. $D$, Maps of time-frequency coherence between the Pre, Pattern, and Post periods for LFPs recorded in the PrL cortex and the other five recording sites. Illustrated maps correspond to the average of 100 samples collected from five different animals. Note the high coherence between recordings collected from PrL and M1 recording sites, mostly for the Pre and Post periods. 
2015), including classical and operant conditioning tasks (LealCampanario et al., 2007; Jurado-Parras et al., 2012). In particular, the effects of the electrical stimulation of the PrL cortex during the operant sequence used here suggest the involvement of this area in ongoing cognitive processes related to the association between touching the screen and getting the corresponding reward-a finding also reported in mice during a similar operant conditioning task (Jurado-Parras et al., 2012).

The behaviors (i.e., remaining motionless, rearing, and looking into the feeder; Fig. 4) during which the selected $\theta / \beta-\gamma$ transition pattern was observed, as well as its intrinsic spectral characteristics (a significant decrease in power of the $\theta$ band associated with smaller changes in the $\beta$ and $\gamma$ bands), could be related to the proposal that LFPs presenting low amplitudes and high- $\gamma$ frequencies are involved in attentive processes (Moruzzi and Magoun, 1949; Fries et al., 2001; Poulet and Petersen, 2008; Zagha et al., 2013) and with specific hypervigilance rhythms associated with immobility and focused attention (Bouyer et al., 1981; Wang, 2010; Koralek et al., 2012). Interestingly, an LFP pattern similar to the one described here has been reported to be present in neocortical S1 and M1 areas during whisking conditions (Zagha et al., 2013). Nevertheless, apart from this similarity, the present $\theta / \beta-\gamma$ transition pattern was more closely related to a significant decrease in the activity of whisker motor responses (Fig. 7). In fact, coherence in LFPs recorded simultaneously in $\operatorname{PrL}$ and $\mathrm{M} 1$ cortices decreases during the presentation of the $\theta / \beta-\gamma$ transition pattern (Fig. 9). Thus, this pattern seems not to be related to specific $\beta$ activities present in the M1 cortex during selected motor responses (Zagha et al., 2013; Khanna and Carmena, 2015).

The learning curve generated by the pattern-evoked cue presented a sigmoidal shape similar to (although with a slower evolution than) that generated by experimental animals during the automatic presentation of the cue on the touch screen (Fig. 6B) or during rewarded lever pressing in the Skinner box (Karni et al., 1998; Buitrago et al., 2004; Jurado-Parras et al., 2012). These results suggest that the use of an LFP pattern generated in the PrL cortex to activate an operant conditioning task does not represent an unviable burden for the experimental animal.

Pattern-evoked cue sessions generated some minor changes in the selected $\theta / \beta-\gamma$ transition pattern, including a progressive increase in pattern duration, a decrease in the power ratio $\left[\mathrm{P}_{\beta}+\right.$ $\left.\mathrm{P}_{\gamma}\right) / \mathrm{P}_{\theta}$; Fig. 5C,D; resulting from faster recovery in the firing rate of PrL neurons], and a significant decrease in the spectral power of $\beta$ and $\gamma$ bands (Fig. $8 H$ ). In fact, these changes increased the probability of activating the touch screen, since a decrease in $\theta+$ $\beta+\gamma$ power increased the likelihood of reaching the selected threshold (Fig. 4). Interestingly, these changes were not observed during the training period in which the cue was presented automatically, a fact suggesting that changes in the selected $\theta / \beta-\gamma$ transition pattern were not due to the association between the cue (square virtual button) and the reward, but by the association between the $\theta / \beta-\gamma$ transition pattern and the activation of the cue on the touch screen. These results coincide with available reports on neuronal operant conditioning in which the reward is obtained with changes in the discharge rate of conditioned neurons (Opris et al., 2011; Arduin et al., 2013). It should be noted that the reported changes in the selected $\theta / \beta-\gamma$ transition pattern observed during patternevoked cue sessions did not modify the main spectral characteristic of the pattern - namely, a significant decrease in $\theta$ and high- $\gamma$ bands.

Interestingly, the selected $\theta / \beta-\gamma$ transition pattern appeared simultaneously with a significant decrease in the mean firing rate of PrL cells (Fig. 6) putatively considered as pyramidal neurons because of their firing properties (Dégenètais et al., 2002; Povysheva et al., 2006; Leal-Campanario et al., 2013). In this regard, it has been recently proposed that the rostromedial prefrontal cortex plays an active role in preventing the release of newly acquired motor responses until advanced stages of the acquisition process and, in general, in the control of the ongoing behavior, depending on environmental and social constraints (Leal-Campanario et al., 2007, 2013). The inhibition of PrL cortex neurons during the $\theta / \beta-\gamma$ transition pattern suggests that this pattern corresponds to a time window for the release of specific behaviors involving cognitive processes (Fig. 4). In addition, the electrical stimulation of the recording area evoked disturbing effects on the animal's performance during the operant task not related to any visible deficit in its motor activities (Hamani et al., 2010). Thus, these results indicate that the stimulation of the PrL cortex disturbed specific cognitive processes underlying the expected behavioral sequences (Jackson et al., 2001; Jurado-Parras et al., 2012).

It is important to point out the low coherence values between LFPs recorded in the PrL cortex, including the selected $\theta / \beta-\gamma$ transition pattern, and LFPs recorded simultaneously in the nucleus accumbens, the motor cortex, the mediodorsal thalamic nucleus, and the hippocampal CA1 and ventral tegmental areas. Apparently, the selected $\theta / \beta$ - $\gamma$ transition pattern was not generated in and/or propagated to the five mentioned areas, but is an activity restricted to the PrL cortex. In fact, we obtained higher $(0.60<R>0.69)$ levels of coherence between LFPs recorded in the PrL cortex compared with those recorded in the motor cortex, the nucleus accumbens, and the mediodorsal thalamic nucleus in the high- $\gamma$ band during the generation of the pattern. In this regard, an increase has been reported in functional interactions in the high- $\gamma$ band between the PrL cortex and the mediodorsal thalamic nucleus during the acquisition of an instrumental conditioning task (Yu et al., 2012), as well as between the nucleus accumbens and the PrL cortex during a similar learning task (Chang et al., 2000). The observation of increased coherence values for narrow frequency bands between different cortical and subcortical areas suggests the involvement of different learning and memory processes taking place at the same time in these different neural structures using the selected $\theta / \beta-\gamma$ transition pattern as a brain-iPad interface.

Finally, it can be questioned whether the $\theta / \beta-\gamma$ transition pattern was just a byproduct of the preferred behavior (sitting still or motionless) used by the experimental rats to activate the iPad. In this regard, and as illustrated in Figure 4, the same LFP pattern was present during the performance of other behaviors (as rearing or looking into the feeder) and, at the same time, was not always present when the animal remained motionless in the cage-immobility per se is not enough to generate this LFP pattern (Gervasoni et al., 2004). Although in early conditioning sessions, animals generated the LFP pattern in different locations within the Skinner box, the animal selected this behavior (sitting still near the iPad) across training because of its adaptive value. In addition, given their rather poor visual acuity (Prusky et al., 2002) being near the iPad will facilitate an early detection of the visual cue. Thus, it can safely be expected that a paralyzed animal should still be able to generate this (and related) LFP patterns without associating it with a given behavior, certain movements, or even adjustments to posture.

\section{References}

Arduin PJ, Frégnac Y, Shulz DE, Ego-Stengel V (2013) “Master” neurons induced by operant conditioning in rat motor cortex during a brainmachine interface task. J Neurosci 33:8308-8320. CrossRef Medline

Bouton CE, Shaikhouni A, Annetta NV, Bockbrader MA, Friedenberg DA, Nielson DM, Sharma G, Sederberg PB, Glenn BC, Mysiw WJ, Morgan AG, Deogaonkar M, Rezai AR (2016) Restoring cortical control of functional movement in a human with quadriplegia. Nature 533:247-250. CrossRef Medline 
Bouyer JJ, Montaron MF, Rougeul A (1981) Fast fronto-parietal rhythms during combined focused attentive behaviour and immobility in cat: cortical and thalamic localizations. Electroencephalogr Clin Neurophysiol 51:244-252. CrossRef Medline

Buitrago MM, Ringer T, Schulz JB, Dichgans J, Luft AR (2004) Characterization of motor skill and instrumental learning time scales in a skilled reaching task in rat. Behav Brain Res 155:249-256. CrossRef Medline

Chang JY, Janak PH, Woodward DJ (2000) Neuronal and behavioral correlations in the medial prefrontal cortex and nucleus accumbens during cocaine selfadministration by rats. Neuroscience 99:433-443. CrossRef Medline

Dégenètais E, Thierry AM, Glowinski J, Gioanni Y (2002) Electrophysiological properties of pyramidal neurons in the rat prefrontal cortex: an in vivo intracellular recording study. Cereb Cortex 12:1-16. CrossRef Medline

Fetz EE (1969) Operant conditioning of cortical unit activity. Science 163: 955-958. CrossRef Medline

Fetz EE, Finocchio DV (1971) Operant conditioning of specific patterns of neural and muscular activity. Science 174:431-435. CrossRef Medline

Fetz EE, Finocchio DV (1972) Operant conditioning of isolated activity in specific muscles and precentral cells. Brain Res 40:19-23. CrossRef Medline

Flint RD, Wright ZA, Scheid MR, Slutzky MW (2013) Long term, stable brain machine interface performance using local field potentials and multiunit spikes. J Neural Eng 10:056005. CrossRef Medline

Freeman WJ, Holmes MD, Burke BC, Vanhatalo S (2003) Spatial spectra of scalp EEG and EMG from awake humans. Clin Neurophysiol 114:105368. CrossRef Medline

Fries P, Reynolds JH, Rorie AE, Desimone R (2001) Modulation of oscillatory neuronal synchronization by selective visual attention. Science 291: 1560-1563. CrossRef Medline

Fuster JM (2015) The prefrontal cortex. London: Academic.

Gervasoni D, Lin SC, Ribeiro S, Soares ES, Pantoja J, Nicolelis MA (2004) Global forebrain dynamics predict rat behavioral states and their transitions. J Neurosci 24:11137-11147. CrossRef Medline

Graybeal C, Feyder M, Schulman E, Saksida LM, Bussey TJ, Brigman JL, Holmes A (2011) Paradoxical reversal learning enhancement by stress or prefrontal cortical damage: rescue with BDNF. Nat Neurosci 14:15071509. CrossRef Medline

Gruart A, Muñoz MD, Delgado-García JM (2006) Involvement of the CA3CA1 synapse in the acquisition of associative learning in behaving mice. J Neurosci 26:1077-1087. CrossRef Medline

Hamani C, Diwan M, Isabella S, Lozano AM, Nobrega JN (2010) Effects of different stimulation parameters on the antidepressant-like response of medial prefrontal cortex deep brain stimulation in rats. J Psychiatr Res 44:683-687. CrossRef Medline

Hasan MT, Hernández-González S, Dogbevia G, Treviño M, Bertocchi I, Gruart A, Delgado-García JM (2013) Role of motor cortex NMDA receptors in learning-dependent synaptic plasticity of behaving mice. Nat Commun 4:2258. CrossRef Medline

Hiremath SV, Chen W, Wang W, Foldes S, Yang Y, Tyler-Kabara EC, Collinger JL, Boninger ML (2015) Brain computer interface learning for systems based on electrocorticography and intracortical microelectrode arrays. Front Integr Neurosci 9:40. CrossRef Medline

Hong KS, Naseer N, Kim YH (2015) Classification of prefrontal and motor cortex signals for three-class fNIRS-BCI. Neurosci Lett 587:87-92. CrossRef Medline

Hoover WB, Vertes RP (2007) Anatomical analysis of afferent projections to the medial prefrontal cortex in the rat. Brain Struct Funct 212:149-179. CrossRef Medline

Jackson ME, Frost AS, Moghaddam B (2001) Stimulation of prefrontal cortex at physiologically relevant frequencies inhibits dopamine release in the nucleus accumbens. J Neurochem 78:920-923. CrossRef Medline

Jacobs GH, Fenwick JA, Williams GA (2001) Cone-based vision of rats for ultraviolet and visible lights. J Exp Biol 204:2439-2446. Medline

Jurado-Parras MT, Gruart A, Delgado-García JM (2012) Observational learning in mice can be prevented by medial prefrontal cortex stimulation and enhanced by nucleus accumbens stimulation. Learn Mem 19:99-106. CrossRef Medline

Kable JW, Glimcher PW (2009) The neurobiology of decision: consensus and controversy. Neuron 63:733-745. CrossRef Medline

Karni A, Meyer G, Rey-Hipolito C, Jezzard P, Adams MM, Turner R, Ungerleider LG (1998) The acquisition of skilled motor performance: fast and slow experience-driven changes in primary motor cortex. Proc Natl Acad Sci U S A 95:861-868. CrossRef Medline
Khanna P, Carmena JM (2015) Neural oscillations: beta band activity across motor networks. Curr Opin Neurobiol 32:60-67. CrossRef Medline

Koralek AC, Jin X, Long JD 2nd, Costa RM, Carmena JM (2012) Corticostriatal plasticity is necessary for learning intentional neuroprosthetic skills. Nature 483:331-335. CrossRef Medline

Leal-Campanario R, Fairén A, Delgado-García JM, Gruart A (2007) Electrical stimulation of the rostral medial prefrontal cortex in rabbits inhibits the expression of conditioned eyelid responses but not their acquisition. Proc Natl Acad Sci U S A 104:11459-11464. CrossRef Medline

Leal-Campanario R, Delgado-García JM, Gruart A (2013) The rostral medial prefrontal cortex regulates the expression of conditioned eyelid responses in behaving rabbits. J Neurosci 33:4378-4386. CrossRef Medline

Lebedev MA, Nicolelis MA (2006) Brain-machine interfaces: past, present and future. Trends Neurosci 29:536-546. CrossRef Medline

Leising KJ, Wolf JE, Ruprecht CM (2013) Visual discrimination learning with an iPad-equipped apparatus. Behav Processes 93:140-147. CrossRef Medline

Mehring C, Rickert J, Vaadia E, Cardosa de Oliveira S, Aertsen A, Rotter S (2003) Inference of hand movements from local field potentials in monkey motor cortex. Nat Neurosci 6:1253-1254. CrossRef Medline

Moruzzi G, Magoun HW (1949) Brain stem reticular formation and activation of the EEG. Electroencephalogr Clin Neurophysiol 1:455-473. CrossRef Medline

O'Doherty JE, Lebedev MA, Ifft PJ, Zhuang KZ, Shokur S, Bleuler H, Nicolelis MA (2011) Active tactile exploration using a brain-machine-brain interface. Nature 479:228-231. CrossRef Medline

Opris I, Lebedev M, Nelson RJ (2011) Motor planning under unpredictable reward: modulations of movement vigor and primate striatum activity. Front Neurosci 5:61. CrossRef Medline

Paxinos G, Watson C (1998) The rat brain in stereotaxic coordinates. San Diego: Academic.

Pesaran B, Pezaris JS, Sahani M, Mitra PP, Andersen RA (2002) Temporal structure in neuronal activity during working memory in macaque parietal cortex. Nat Neurosci 5:805-811. CrossRef Medline

Poulet JF, Petersen CC (2008) Internal brain state regulates membrane potential synchrony in barrel cortex of behaving mice. Nature 454:881-885. CrossRef Medline

Povysheva NV, Gonzalez-Burgos G, Zaitsev AV, Kröner S, Barrionuevo G, Lewis DA, Krimer LS (2006) Properties of excitatory synaptic responses in fast-spiking interneurons and pyramidal cells from monkey and rat prefrontal cortex. Cereb Cortex 16:541-552. CrossRef Medline

Prusky GT, Harker KT, Douglas RM, Whishaw IQ (2002) Variation in visual acuity within pigmented, and between pigmented and albino rat strains. Behav Brain Res 136:339-348. CrossRef Medline

Rouse AG, Williams JJ, Wheeler JJ, Moran DW (2013) Cortical adaptation to a chronic micro-electrocorticographic brain computer interface. J Neurosci 33:1326-1330. CrossRef Medline

Schafer RJ, Moore T (2011) Selective attention from voluntary control of neurons in prefrontal cortex. Science 332:1568-1571. CrossRef Medline

Schudlo LC, Power SD, Chau T (2013) Dynamic topographical pattern classification of multichannel prefrontal NIRS signals. J Neural Eng 10: 046018. CrossRef Medline

Shain W, Spataro L, Dilgen J, Haverstick K, Retterer S, Isaacson M, Saltzman M, Turner JN (2003) Controlling cellular reactive responses around neural prosthetic devices using peripheral and local intervention strategies. IEEE Trans Neural Syst Rehabil 11:186-188. CrossRef Medline

So K, Dangi S, Orsborn AL, Gastpar MC, Carmena JM (2014) Subject-specific modulation of local field potential spectral power during brain-machine interface control in primates. J Neural Eng 11:026002. CrossRef Medline

Vertes RP (2004) Differential projections of the infralimbic and prelimbic cortex in the rat. Synapse 51:32-58. CrossRef Medline

Wander JD, Blakely T, Miller KJ, Weaver KE, Johnson LA, Olson JD, Fetz EE, Rao RP, Ojemann JG (2013) Distributed cortical adaptation during learning of a brain-computer interface task. Proc Natl Acad Sci U S A 110:10818-10823. CrossRef Medline

Wang XJ (2010) Neurophysiological and computational principles of cortical rhythms in cognition. Physiol Rev 90:1195-1268. CrossRef Medline

Yu C, Fan D, Lopez A, Yin HH (2012) Dynamic changes in single unit activity and $\gamma$ oscillations in a thalamocortical circuit during rapid instrumental learning. PLoS One. 7:e50578. CrossRef Medline

Zagha E, Casale AE, Sachdev RN, McGinley MJ, McCormick DA (2013) Motor cortex feedback influences sensory processing by modulating network state. Neuron 79:567-578. CrossRef Medline 